Homology, Homotopy and Applications, vol.19(2), 2017, pp.199-218

\title{
A NOTE ON THE ALGEBRAIC DE RHAM UNIVERSAL CLASSES
}

\section{MAREK GOLASIŃSKI AND FRANCISCO GÓMEZ RUIZ}

\author{
(communicated by Aleksander Mishchenko)
}

\begin{abstract}
This paper contains the algebraic analog of universal classifying bundles and Chern classes. We imitate the topological counterpart of universal bundles over the Grassmannian to construct some graded commutative differential algebras $\hat{\Omega}^{*}\left(\hat{K}[X] /\left(X^{2}-X, \operatorname{tr} X-r\right)\right)$ and $\hat{\Omega}^{*}\left(\hat{K}[X] /\left(X^{2}-X\right)\right)$, whose corresponding cohomology are polynomial algebras isomorphic to $K\left[\bar{c}_{1}, \ldots, \bar{c}_{r}\right]$ and $K\left[\bar{c}_{1}, \bar{c}_{2}, \ldots\right]$ respectively, for the Chern classes $\bar{c}_{p}$ with $p \geqslant 1$, for the field $K=\mathbb{Q}, \mathbb{R}$ or $\mathbb{C}$. Here $X$ denotes the infinite matrix $X=\left[X_{p q}\right], X^{n}$ denotes the corresponding matrix obtained from $X$ by setting to zero the entries $X_{p q}$ when $p>n$ or $q>n$, and $\left(X^{2}-X, \operatorname{tr} X-r\right)\left(\right.$ resp. $\left.\left(X^{2}-X\right)\right)$ denotes the ideal generated by the power series $\sum_{p} X_{p p}-r$ and the entries of the matrix $X^{2}-X$ (resp. the entries of $\left.X^{2}-X\right)$.
\end{abstract}

\section{Introduction}

It is well known, after Swan [9], the equivalence between isomorphism classes of vector bundles and that of finitely generated projective modules over the ring of continuous functions on the base or, equivalently, idempotent matrices over the ring of those functions.

Given a smooth complex vector bundle $\xi$, represented by an idempotent matrix $\varphi$ of order $n$, over the ring of smooth functions on the base, the Chern classes of $\xi$ in the standard de Rham cohomology of differential forms, are given as follows:

$$
\operatorname{det}\left(I_{n}+\varphi(d \varphi)^{2}\right)=1+c_{1}\left(\varphi\left(d(\varphi)^{2}\right)\right)+\cdots+c_{n}\left(\varphi\left(d(\varphi)^{2}\right)\right),
$$

where $c_{p}$ is the $p$-th characteristic coefficient for $p=1, \ldots, n$. Then, $c_{p}\left(\varphi(d \varphi)^{2}\right)$ is a closed form and $\frac{1}{(-2 \pi i)^{p}} c_{p}\left(\varphi(d \varphi)^{2}\right)$ represents the $p$-th Chern class of $\xi$.

Complex vector bundles of rank $r$ are classified by free homotopy classes of maps from the base space to the Grassmannian $G_{-, r}(\mathbb{C})$ over the field $\mathbb{C}$ of complex numbers whose points are the $r$-dimensional vector subspaces of $\mathbb{C}^{\infty}$. Consider the smooth manifolds Idem $_{n, r}(\mathbb{C})$ of idempotent $n \times n$-matrices of rank $r$ over $\mathbb{C}$ and the union $\operatorname{Idem}_{-, r}(\mathbb{C})=\cup_{n \geqslant r} \operatorname{Idem}_{n, r}(\mathbb{C})$.

This research was partially supported by MEC-FEDER grant MTM2013-41768-P and JA grants FQM-213.

Received January 20, 2017, revised February 22, 2017; published on November 15, 2017.

2010 Mathematics Subject Classification: Primary 14F40, 55R40; secondary 19A49.

Key words and phrases: algebraic de Rham cohomology, Grassmannian, idempotent matrix, universal Chern class.

Article available at http://dx.doi.org/10.4310/HHA.2017.v19.n2.a11

Copyright (C) 2017, International Press. Permission to copy for private use granted. 
The algebraic translation of the classifying space and classifying map for vector bundles would be, in general, to consider the quotient ring $\mathbb{Z}[[X]] /\left(X^{2}-X\right)$, where $\mathbb{Z}[[X]]$ is the formal power series ring over the integers $\mathbb{Z}$ with indeterminates $X_{p q}$ for $p, q \geqslant 1$ and $\left(X^{2}-X\right)$ denotes the ideal generated by the entries of the matrix $\left(X^{2}-X\right)$ with $X=\left(X_{p q}\right)$. We say that $X$ is the universal idempotent matrix. In particular, it represents a class in the Grothendieck group $\tilde{K}_{0}\left(\mathbb{Z}[[X]] /\left(X^{2}-X\right)\right)$ and has universal Chern classes $\bar{c}_{p}(X)$ with respect to the algebraic de Rham complex over the ring $\mathbb{Z}[[X]] /\left(X^{2}-X\right)$, where $\bar{c}_{p}(X) \in H_{d R}^{2 p}\left(\mathbb{Z}[[X]] /\left(X^{2}-X\right)\right)$.

The analog of a classifying map for a given idempotent matrix $\varphi=\left(\varphi_{p q}\right)$ with entries in some commutative ring $\mathcal{A}$ is then the canonical ring homomorphism $\varphi: \mathbb{Z}[[X]] /\left(X^{2}-X\right) \rightarrow \mathcal{A}$ given by sending the class of $X_{p q}$ to $\varphi_{p q}$. Therefore, we have a ring homomorphism $\varphi^{*}: \tilde{K}_{0}\left(\mathbb{Z}[[X]] /\left(X^{2}-X\right)\right) \rightarrow \tilde{K}_{0}(\mathcal{A})$, which, in particular, sends the class of $X$ to the class of $\varphi$.

As a particular case of Grothendieck [5] results we have the canonical isomorphism

$$
H_{d R}^{*}\left(\mathbb{C}\left[X^{n}\right] /\left(\left(X^{n}\right)^{2}-X^{n}, \operatorname{tr} X^{n}-r\right)\right) \stackrel{\simeq}{\longrightarrow} H_{d R}^{*}\left(\operatorname{Idem}_{n, r}(\mathbb{C})\right),
$$

where $X^{n}$ is the $n \times n$-matrix with entries $X_{p q}$ for $1 \leqslant p, q \leqslant n$. Then, the paper [4] contains an algebraic analog for idempotent matrices of the Chern-Weil theory of characteristic classes.

We were inspired by those counterparts to construct, for any field of characteristic zero $K$, commutative differential algebras $\hat{\Omega}^{*}\left(\hat{K}[X] /\left(X^{2}-X, \operatorname{tr} X-r\right)\right)$ and $\hat{\Omega}^{*}\left(\hat{K}[X] /\left(X^{2}-X\right)\right)$, with $X=\left[X_{p q}\right]$ and $p, q \geqslant 1$, where $\hat{K}[X]$ is a subring of the formal power series ring $K[[X]]$, to show that their cohomology algebras are isomorphic to $K\left[\bar{c}_{1}, \ldots, \bar{c}_{r}\right]$ and $K\left[\bar{c}_{1}, \bar{c}_{2}, \ldots\right]$, respectively for the universal Chern characteristic classes $\bar{c}_{p}$ with $p \geqslant 1$.

In Section 1, we set the stage for developments to come. This introductory section is devoted to a general discussion and establishes notation on the Kähler differentials $\Omega_{R}(\mathcal{A})$ of an $R$-algebra $\mathcal{A}$ and the associated de Rham complex $\Omega_{R}^{*}(\mathcal{A})$ used in the rest of the paper.

Section 2 is based mainly on [4] and is devoted to a list of standard results on algebraic characteristic classes for idempotent matrices.

The aim of Section 3 is to show $G_{n, r}(\mathbb{C})$ is dense in $\operatorname{Idem}_{n, r}(\mathbb{C})$ with respect to the Zariski topology on the affine space $M_{n}(\mathbb{C}) \simeq \mathbb{C}^{n^{2}}$.

Write $\operatorname{Pol}_{K}\left(\operatorname{Idem}_{n, r}(\mathbb{C})\right)$ for the subalgebra of polynomial functions on $\operatorname{Idem}_{n, r}(\mathbb{C})$ with coefficients in $K$, generated by the projection maps $x_{p q}: \operatorname{Idem}_{n, r}(\mathbb{C}) \rightarrow \mathbb{C}$. Then, our main results are:

Theorem 4.7. We have $H_{d R}^{*}\left(K\left[X^{n}\right] /\left(\left(X^{n}\right)^{2}-X^{n}, \operatorname{tr} X^{n}-r\right)\right)=K\left[\bar{c}_{1}, \ldots, \bar{c}_{r}\right] / I_{n, r}$, where $\bar{c}_{j} \in H_{d R}^{2 j}\left(K\left[X^{n}\right] /\left(\left(X^{n}\right)^{2}-X^{n}, \operatorname{tr} X^{n}-r\right)\right)$ is the class of the $j$-th Chern form $c_{j}\left(X^{n}\left(d X^{n}\right)^{2}\right) \in \Lambda^{2 j} \Omega^{1}\left(K\left[X^{n}\right] /\left(\left(X^{n}\right)^{2}-X^{n}, \operatorname{tr} X^{n}-r\right)\right)$, and $I_{n, r}$ is the ideal generated by the well known polynomials deduced from the relation $\left(1+c_{1}(\gamma)+\cdots+\right.$ $\left.c_{r}(\gamma)\right)\left(1+c_{1}\left(\gamma^{\perp}\right)+\cdots+c_{n-r}\left(\gamma^{\perp}\right)\right)=1$, where $c_{j}(\gamma)$, resp. $c_{j}\left(\gamma^{\perp}\right)$, is the $j$-th Chern class of the canonical bundle $\gamma$, resp. normal to the canonical bundle $\gamma^{\perp}$, over $G_{n, r}(\mathbb{C})$. (See, e.g., [1, Proposition 23.2].)

and 
Theorem 4.11. There are isomorphisms

$$
\begin{aligned}
H^{*}\left(\operatorname { l i m } _ { n } \left(\Omega_{d R}^{*}(\right.\right. & \left.\left.\left.K\left[X^{n}\right] /\left(\left(X^{n}\right)^{2}-X^{n}, \operatorname{tr} X^{n}-r\right), d\right)\right)\right) \\
& \simeq \lim _{n} H_{d R}^{*}\left(K\left[X^{n}\right] /\left(\left(X^{n}\right)^{2}-X^{n}, \operatorname{tr} X^{n}-r\right)\right) \\
& \simeq H_{d R}^{*}\left(\operatorname{Pol}_{K}\left(\operatorname{Idem}_{-, r}(\mathbb{C})\right)\right) \stackrel{\simeq}{\longrightarrow} H^{*}\left(\operatorname{Idem}_{-, r}(\mathbb{C}) ; K\right)=K\left[\bar{c}_{1}, \ldots, \bar{c}_{r}\right]
\end{aligned}
$$

and

$$
\begin{aligned}
H^{*}\left(\lim _{r} \lim _{n}\left(\Omega_{d R}^{*}\left(K\left[X^{n}\right] /\left(\left(X^{n}\right)^{2}-X^{n}, \operatorname{tr} X^{n}-r\right), d\right)\right)\right) \\
\stackrel{\simeq}{\longrightarrow} \lim _{r} \lim _{n} H_{d R}^{*}\left(K\left[X^{n}\right] /\left(\left(X^{n}\right)^{2}-X^{n}, \operatorname{tr} X^{n}-r\right)\right) \\
\stackrel{\simeq}{\longrightarrow} H_{d R}^{*}\left(\operatorname{Pol}_{K}(\bar{B} U(\mathbb{C}))\right) \stackrel{\simeq}{\longrightarrow} H^{*}(\bar{B} U(\mathbb{C}) ; K)=K\left[\bar{c}_{1}, \bar{c}_{2}, \ldots\right] .
\end{aligned}
$$

Finally, we notice that for a subring $\hat{K}[X]$ of the formal power series ring $K[[X]]$, the quotient ring $\hat{K}[X] /\left(X^{2}-X, \operatorname{tr} X-r\right)$ and the associated complex $\left(\hat{\Omega}^{*}(\hat{K}[X] /\right.$ $\left.\left.\left(X^{2}-X, \operatorname{tr} X-r\right)\right), d\right)$ are isomorphic respectively to the ring of polynomial functions on Idem,$- r(\mathbb{C})$ with $K$-coefficients and to de Rham polynomial forms on $\operatorname{Idem}_{-, r}(\mathbb{C})$ with coefficients in one of the fields $K=\mathbb{Q}, \mathbb{R}$ or $\mathbb{C}$.

The main results of Section 5 are stated in Theorems 5.3-5.4:

The cohomology algebras of $\hat{\Omega}^{*}\left(\hat{K}[X] /\left(X^{2}-X, \operatorname{tr} X-r\right)\right)$ and $\hat{\Omega}^{*}\left(\hat{K}[X] /\left(X^{2}-X\right)\right)$ are isomorphic to $K\left[\bar{c}_{1}, \ldots, \bar{c}_{r}\right]$ and $K\left[\bar{c}_{1}, \bar{c}_{2}, \ldots\right]$, respectively for the universal Chern classes $\bar{c}_{p}$ with $p \geqslant 1$.

As noted in Remark 5.5, it would be interesting to give proofs of our main results that use algebraic tools only.

\section{Prerequisites}

Notice that the Grassmannian $G_{-, r}(\mathbb{C})$ is the union of an increasing sequence of compact smooth manifolds $G_{n, r}(\mathbb{C})$, where the elements of $G_{n, r}(\mathbb{C})$ are the $r$ dimensional subspaces of $\mathbb{C}^{n}$ for $n \geqslant r$.

Let $\operatorname{Herm}(\mathbb{C})$ (resp. $\operatorname{Herm}_{n}(\mathbb{C})$ ) be the real vector space of hermitian matrices (resp. $n \times n$-hermitian matrices) over $\mathbb{C}$. It is clear that $G_{n, r}(\mathbb{C})\left(\operatorname{resp} . G_{-, r}(\mathbb{C})\right.$ ) can be identified with the subset of $\operatorname{Herm}_{n}(\mathbb{C})$ (resp. Herm $(\mathbb{C})$ ) of hermitian and idempotent complex matrices of rank $r$ (resp. of any rank).

Next, write $M(\mathbb{C})$ (resp. $M_{n}(\mathbb{C})$ ) for the complex vector space of matrices over $\mathbb{C}$ (resp. $n \times n$-matrices over $\mathbb{C})$. It is clear that $\operatorname{Idem}_{n, r}(\mathbb{C})\left(\operatorname{resp} . \operatorname{Idem}_{-, r}(\mathbb{C})\right)$ can be identified with the subset of $M_{n}(\mathbb{C})$ (resp. of $M(\mathbb{C})$ ) of idempotent matrices of rank $r$ (resp. of any rank).

In view of [2, Corollary 1.3], or its generalization [3, Theorem 2.3], the Grassmannian $G_{-, r}(\mathbb{C})$ is a strong deformation retract of $\operatorname{Idem}_{-, r}(\mathbb{C})$ with the retraction map $\operatorname{Idem}_{-, r}(\mathbb{C}) \rightarrow G_{-, r}(\mathbb{C})$, given by $\varphi \mapsto \varphi\left(\varphi+\bar{\varphi}^{t}-I_{n}\right)^{-1}$ for $\varphi \in \operatorname{Idem}_{-, r}(\mathbb{C})$.

It is clear that the above retraction restricts to retractions $\operatorname{Idem}_{n, r}(\mathbb{C}) \rightarrow G_{n, r}(\mathbb{C})$ for $n \geqslant 1$. Therefore, up to homotopy, we may replace $G_{-, r}(\mathbb{C})\left(\operatorname{resp} . G_{n, r}(\mathbb{C})\right)$ by $\operatorname{Idem}_{-, r}(\mathbb{C})\left(\right.$ resp. $\left.\operatorname{Idem}_{n, r}(\mathbb{C})\right)$.

As a consequence, Idem,$- r(\mathbb{C})$ also satisfies the property that for an $r$-complex vector bundle over a compact space $B$ there is a map $f: B \rightarrow \operatorname{Idem}_{n, r}(\mathbb{C}) \subset \operatorname{Idem}_{-, r}(\mathbb{C})$ for $n$ enough big, uniquely determined up to free homotopy. 
In this way the complex vector bundle can be identified with the homotopy class of the map $f$ and then with the class in the Grothendieck group $\tilde{K}_{0}(B)$ of idempotent $n \times n$-matrices over the ring of complex continuous function on $\operatorname{Idem}_{n, r}(\mathbb{C})$. We simply identify $f$ with the matrix whose $(i, j)$-entry is the map $f_{i j}: B \rightarrow \mathbb{C}$, where $f_{i j}(b)$ is the $(i, j)$-entry of $f(b)$ for $b \in B$.

In the particular case of $\xi$ being the universal complex vector bundle over Idem $_{-, r}(\mathbb{C})$ whose fiber over $A \in \operatorname{Idem}_{-, r}(\mathbb{C})$ is the image of $A$, the map $f$ above is certainly the identity map on $\operatorname{Idem}_{-, r}(\mathbb{C})$.

Therefore, the universal complex vector bundle over $\operatorname{Idem}_{-, r}(\mathbb{C})$ can be identified with the infinite idempotent matrix $X$ whose $(p, q)$-th entry is the restriction to Idem $_{-, r}(\mathbb{C})$ of the projection map $x_{p q}: M(\mathbb{C}) \rightarrow \mathbb{C}$ determined by the $(p, q)$-th entry.

Although Idem,$- r(\mathbb{C})$ is not a smooth manifold, being the union of the increasing sequence of manifolds $\operatorname{Idem}_{n, r}(\mathbb{C})$ for $n \geqslant r$, it makes sense to consider the standard de Rham complex of smooth differential forms on $\operatorname{Idem}_{-, r}(\mathbb{C})$.

We also consider the sequence of inclusions $\tau_{r}$ : $\operatorname{Idem}_{-, r}(\mathbb{C}) \hookrightarrow \operatorname{Idem}_{-, r+1}(\mathbb{C})$, given by

$$
\tau_{r}(A)=\left(\begin{array}{ll}
1 & 0 \\
0 & A
\end{array}\right) \text { for } A \in \operatorname{Idem}_{-, r}(\mathbb{C})
$$

and the colimit

$$
\bar{B} U(\mathbb{C})=\operatorname{colim}_{r} \operatorname{Idem}_{-, r}(\mathbb{C}) .
$$

Now, for an idempotent $n \times n$-matrix $\varphi=\left(\varphi_{p q}\right)$ with entries in an $R$-algebra $\mathcal{A}$ and a commutative graded differential algebra $(\Omega, d)$ with $\Omega^{0}=\mathcal{A}$ then we write

$$
\operatorname{det}\left(I_{n}+\varphi(d \varphi)^{2}\right)=\sum_{k \geqslant 0} c_{p}(\varphi)
$$

and so we obtain closed $2 p$-forms $c_{p}(\varphi) \in \Omega^{2 p}$, and the corresponding $2 p$-cohomology classes $\bar{c}_{p}(\varphi) \in H^{2 p}(\Omega, d)$.

We say that $c_{p}(\varphi)$ (resp. $\left.\bar{c}_{p}(\varphi)\right)$ is the $p$-th Chern form (resp. $p$-th Chern class) of the idempotent matrix $\varphi$ with respect to the commutative graded differential algebra $(\Omega, d)$. We also say that $c(\varphi)=\sum_{p \geqslant 0} c_{p}(\varphi)\left(\operatorname{resp} . \bar{c}(\varphi)=\sum_{p \geqslant 0} \bar{c}_{p}(\varphi)\right)$, is the total (non-homogeneous) Chern form (resp. the total Chern class) of the idempotent matrix $\varphi$ with respect to $(\Omega, d)$.

The main example for such a graded commutative algebra is provided by the algebraic de Rham complex of an $R$-algebra. We make use of [7] to recall this construction used in the sequel. Let $\mathcal{A}$ be an algebra over a commutative ring with unit $R$ and $\Omega_{R}(\mathcal{A})=I / I^{2}$ is the $\mathcal{A}$-module of Kähler differentials, where $I$ is the kernel of the multiplication $\mathcal{A} \otimes \mathcal{A} \rightarrow \mathcal{A}$. Given $a \in \mathcal{A}$, write $d a$ for the class of $a \otimes 1-1 \otimes a$ in $\Omega_{R}(\mathcal{A})$. Further $\Omega_{R}^{p}(\mathcal{A})$ is the $p$-exterior power of $\Omega_{R}(\mathcal{A})$ and the derivation $d: \mathcal{A} \rightarrow \Omega_{R}(\mathcal{A})$ extends to a differential of degree 1 . We say that $\left(\Omega_{R}^{*}(\mathcal{A}), d\right)$ is the algebraic de Rham complex of $\mathcal{A}$. The cohomology of this complex is the algebraic de Rham cohomology of $\mathcal{A}$ denoted by $H_{d R}^{*}(\mathcal{A})$.

In the sequel we need the following (probably well known to experts) next two lemmas. Let $i: R \rightarrow R^{\prime}$ be a homomorphism of commutative rings with unit, regard $R^{\prime}$ as an $R$-algebra, and let $\mathcal{A}$ be an $R$-algebra. In particular, we also have the $R^{\prime}$ algebra $R^{\prime} \otimes_{R} \mathcal{A}$. 
Lemma 1.1. The $R^{\prime} \otimes_{R} \mathcal{A}$-modules $\Omega_{R^{\prime}}\left(R^{\prime} \otimes_{R} \mathcal{A}\right)$ and $R^{\prime} \otimes_{R} \Omega_{R}(\mathcal{A})$ are canonically isomorphic.

Proof. Let $\Omega(\varphi): \Omega_{R}(\mathcal{A}) \rightarrow \Omega_{R}\left(R^{\prime} \otimes_{R} \mathcal{A}\right)$ be the $\mathcal{A}$-module homomorphism induced by the canonical homomorphism $\varphi: \mathcal{A} \rightarrow R^{\prime} \otimes_{R} \mathcal{A}$.

Define

$$
\bar{\varphi}: R^{\prime} \otimes_{R} \Omega_{R}(\mathcal{A}) \rightarrow \Omega_{R}\left(R^{\prime} \otimes_{R} \mathcal{A}\right)
$$

by $\bar{\varphi}(\lambda \otimes \omega)=(\lambda \otimes 1) \Omega(\varphi)(\omega)$.

It is clear that $\bar{\varphi}$ is a well defined $R^{\prime} \otimes_{R} \mathcal{A}$-module homomorphism.

On the other hand, observe that $1 \otimes(i \circ d): R^{\prime} \otimes_{R} \mathcal{A} \rightarrow R^{\prime} \otimes_{R} \Omega_{R} \mathcal{A}$ is a derivation on the $R^{\prime} \otimes_{R} \mathcal{A}$-module $R^{\prime} \otimes_{R} \Omega_{R} A$. Then the universal property [7, Proposition, p. 182] for Kähler differentials yields a unique $R^{\prime} \otimes_{R} \mathcal{A}$-module homomorphism $\psi: \Omega_{R^{\prime}}\left(R^{\prime} \otimes \mathcal{A}\right) \rightarrow R^{\prime} \otimes_{R} \Omega_{R} \mathcal{A}$, such that $\psi(d(1 \otimes a))=1 \otimes a$ for $a \in \mathcal{A}$.

Finally, it is clear that $\bar{\varphi}$ and $\psi$ are mutually inverse.

We keep the notation of Lemma 1.1 to show:

Lemma 1.2. There is an isomorphism $H_{d R}^{*}\left(R^{\prime} \otimes_{R} \mathcal{A}\right) \rightarrow H^{*}\left(R^{\prime} \otimes_{R} \Omega_{R}^{*}(\mathcal{A}), 1 \otimes d\right)$. If $R^{\prime}$ is $R$-flat then the canonical map $R^{\prime} \otimes_{R} H_{d R}^{*}(\mathcal{A}) \rightarrow H_{d R}^{*}\left(R^{\prime} \otimes_{R} A\right)$ is an isomorphism.

Proof. The canonical isomorphism $\Omega_{R^{\prime}}\left(R^{\prime} \otimes_{R} \mathcal{A}\right) \rightarrow R^{\prime} \otimes_{R} \Omega_{R} \mathcal{A}$ clearly extends to an isomorphism of graded differential algebras

$$
\left(\Omega_{R^{\prime}}^{*}\left(R^{\prime} \otimes_{R} \mathcal{A}\right), d\right) \rightarrow\left(R^{\prime} \otimes_{R} \Omega_{R}^{*}(\mathcal{A}), 1 \otimes d\right)
$$

and this yields the first isomorphism.

If $R^{\prime}$ is $R$-flat then the Künneth formula leads to an isomorphism

$$
R^{\prime} \otimes_{R} H_{d R}^{*} \mathcal{A} \rightarrow H^{*}\left(R^{\prime} \otimes_{R} \Omega_{R}^{*}(\mathcal{A}), 1 \otimes d\right)
$$

and this concludes the proof.

Let now $\mathcal{A}$ be an algebra over a commutative ring with unit $R$ and $\left(\Omega_{n}(\mathcal{A}), \delta_{n}\right)$ a sequence of cochain complexes over $\mathcal{A}$. Suppose that there are $\mathcal{A}$-module epimorphisms

$$
\rho_{n}^{n+1}: \Omega_{n+1}(\mathcal{A}) \rightarrow \Omega_{n}(\mathcal{A}) .
$$

Then we get the inverse limit cochain complex

$$
(\Omega(\mathcal{A}), \delta)=\lim _{n}\left(\Omega_{n}(\mathcal{A}), \delta_{n}\right) .
$$

We say that the Mittag-Leffler condition is satisfied if there exists a $k$ such that

$$
H^{q-1}\left(\Omega_{n+1}(\mathcal{A}), \delta_{n+1}\right) \rightarrow H^{q-1}\left(\Omega_{n}(\mathcal{A}), \delta_{n}\right)
$$

is an epimorphism for all $n \geqslant k$ and all $q \geqslant 1$.

Then, in view of [10, Theorem 3.5.8] we have the following:

Lemma 1.3. The canonical homomorphism $\varphi: H^{q}(\Omega(\mathcal{A}), \delta) \rightarrow \lim _{n} H^{q}\left(\Omega_{n}(\mathcal{A}), \delta_{n}\right)$ is an epimorphism. It is an isomorphism provided the Mittag-Leffler condition is satisfied. 


\section{Algebraic characteristic classes for idempotent matrices}

Let $R$ be a commutative ring with unit, $(\Omega, d)$ a commutative graded differential algebra over $R$, where $\Omega=\oplus_{k} \geqslant 0 \Omega^{k}$ and write $\Omega^{0}=\mathcal{A}$.

Suppose now that $\varphi$ is an idempotent $n \times n$-matrix with entries in $\mathcal{A}$. Then we make use of [4] to show that the following lemmas hold:

Lemma 2.1. (1) $\varphi(d \varphi) \varphi=0$;

(2) $\varphi(d \varphi)^{2 p}=(d \varphi)^{2 p} \varphi, p \geqslant 1$;

(3) $\operatorname{tr}\left(\varphi(d \varphi)^{2 p+1}\right)=0, p \geqslant 0$.

Proof. (1): Because $\varphi^{2}=\varphi$, we derive $\varphi(d \varphi)+(d \varphi) \varphi=d \varphi$ and $\varphi(d \varphi) \varphi+(d \varphi) \varphi=$ $(d \varphi) \varphi$. Hence $\varphi(d \varphi) \varphi=0$.

(2): The relation $\varphi(d \varphi)+(d \varphi) \varphi=d \varphi$ leads to $(d \varphi)^{2}=\varphi(d \varphi)^{2}+(d \varphi) \varphi(d \varphi)=$ $(d \varphi) \varphi(d \varphi)+(d \varphi)^{2} \varphi$. Therefore $\varphi(d \varphi)^{2}=(d \varphi)^{2} \varphi$ and so

$$
\varphi(d \varphi)^{2 p}=(d \varphi)^{2 p} \varphi \text { for } p \geqslant 1 .
$$

(3): Notice that $\operatorname{tr}\left(\varphi(d \varphi)^{2 p+1}\right)=\operatorname{tr}\left(\varphi(d \varphi)^{2 p+1} \varphi\right)$. Then, using (2) and (1) we get

$$
\operatorname{tr}\left(\varphi(d \varphi)^{2 p+1}\right)=\operatorname{tr}\left((d \varphi)^{2 p} \varphi(d \varphi) \varphi\right)=0 \text { for } p \geqslant 0
$$

and the proof follows.

Lemma 2.2. (1) $(d \varphi)^{p}=0$ for $p>n^{2}$;

(2) $I_{n}+\varphi(d \varphi)^{2}$ is invertible with $\left(I_{n}+\varphi(d \varphi)^{2}\right)^{-1}=\sum_{k \geqslant 0}(-1)^{k}\left(\varphi(d \varphi)^{2}\right)^{k}$, where $I_{n}$ is the identity $n \times n$-matrix.

Lemma 2.3. $d\left(\operatorname{det}\left(I_{n}+\varphi(d \varphi)^{2}\right)\right)=0$.

Proof. We clearly have

$$
d\left(\operatorname{det}\left(I_{n}+\varphi(d \varphi)^{2}\right)\right)=\operatorname{tr}\left(\operatorname{Adj}\left(I_{n}+\varphi(d \varphi)^{2}\right)^{t}(d \varphi)^{3}\right),
$$

where Adj means the adjoint matrix operation.

Therefore

$$
d\left(\operatorname{det}\left(I_{n}+\varphi(d \varphi)^{2}\right)\right)=\operatorname{det}\left(I_{n}+\varphi(d \varphi)^{2}\right) \operatorname{tr}\left(\left(I_{n}+\varphi(d \varphi)^{2}\right)^{-1}(d \varphi)^{3}\right)
$$

and we conclude the proof by using Lemmas 2.1 and 2.2 .

Recall that idempotent matrices $\varphi$ and $\psi$ are said to be equivalent whenever there are matrices $a$ and $b$ such that $a b=\varphi$ and $b a=\psi$. Therefore $\varphi$ and $\psi$ represent the same class in the group $\tilde{K}_{0}(\mathcal{A})$. Then [4, Proposition] yields:

Proposition 2.4. If $\varphi$ and $\psi$ are equivalent idempotent matrices then $\bar{c}_{p}(\varphi)=\bar{c}_{p}(\psi)$ for $p \geqslant 1$.

In particular, we have a well defined map $\bar{c}: \tilde{K}_{0}(\mathcal{A}) \rightarrow H^{\text {even }}(\Omega, d)$.

Now, consider the quotient $\operatorname{ring} \mathbb{Z}[[X]] /\left(X^{2}-X\right)$, where $\mathbb{Z}[[X]]$ is the formal power series ring over the integers $\mathbb{Z}$ with indeterminates $X_{p q}$ for $p, q \geqslant 1$ and $\left(X^{2}-X\right)$ denotes the ideal generated by the entries of the matrix $\left(X^{2}-X\right)$ with $X=\left(X_{p q}\right)$. We say that $X$ is the universal idempotent matrix. In particular, it represents a class in the Grothendieck group $\tilde{K}_{0}\left(\mathbb{Z}[[X]] /\left(X^{2}-X\right)\right)$ and has universal Chern classes $\bar{c}_{p}(X)$ 
with respect to the algebraic de Rham complex over the ring $\mathbb{Z}[[X]] /\left(X^{2}-X\right)$, where $\bar{c}_{p}(X) \in H_{d R}^{2 p}\left(\mathbb{Z}[[X]] /\left(X^{2}-X\right)\right)$. We may regard now the polynomial matrix $X$ as a matrix with entries in the quotient ring $\mathbb{Z}[[X]] /\left(X^{2}-X\right)$ and so $X$ is an idempotent matrix. This is the algebraic counterpart of the universal complex vector bundle.

A matrix $\varphi=\left(\varphi_{p q}\right)$ with entries in an $R$-algebra $\mathcal{A}$ yields the canonical ring homomorphism $\varphi: \mathbb{Z}[[X]] /\left(X^{2}-X\right) \rightarrow \mathcal{A}$ given by sending the class of $X_{p q}$ to $\varphi_{p q}$ if $1 \leqslant p, q \leqslant n$ and to zero, otherwise. Therefore, we have a ring homomorphism $\varphi^{*}: \tilde{K}_{0}\left(\mathbb{Z}[[X]] /\left(X^{2}-X\right)\right) \rightarrow \tilde{K}_{0}(\mathcal{A})$, which, in particular, sends the class of $X$ to the class of $\varphi$.

Further, we have a ring homomorphism $\varphi^{*}: H_{d R}^{*}\left(\mathbb{Z}[[X]] /\left(X^{2}-X\right)\right) \rightarrow H^{*}(\Omega, d)$ sending the universal Chern classes $\bar{c}_{p}(X)$ to the Chern classes $\bar{c}_{p}(\varphi)$ with respect to $(\Omega, d)$ and such that the following diagram

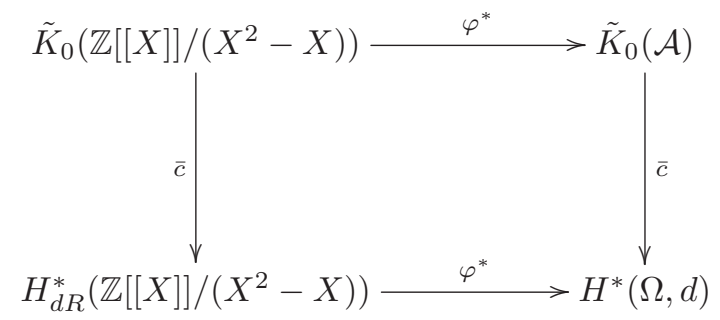

commutes.

For natural numbers $r, \alpha, \beta$ with $r \geqslant 0, \alpha \geqslant 1, \beta \geqslant 1$, we define maps

$$
x_{\alpha \beta}: \operatorname{Idem}_{-, r}(\mathbb{C}) \rightarrow \mathbb{C},
$$

by $x_{\alpha \beta}(A)=a_{\alpha \beta}$ provided $A=\left[a_{p q}\right] \in \operatorname{Idem}_{-, r}(\mathbb{C})$.

These maps are smooth in the sense that they are so once restricted to any smooth

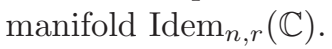

It makes sense to consider smooth forms on $\operatorname{Idem}_{-, r}(\mathbb{C})$ and then on $\bar{B} U(\mathbb{C})$, i.e., a p-form $\Phi$ on $\bar{B} U(\mathbb{C})$ is a sequence of smooth p-forms $\Phi_{r}$ on Idem ${ }_{-, r}(\mathbb{C})$ such that $\tau^{*}\left(\Phi_{r+1}\right)=\Phi_{r}$. Therefore we have the canonical extension to a de Rham complex on $\bar{B} U(\mathbb{C})$.

In particular, $X$ can be regarded as a matrix of complex smooth functions on $\bar{B} U(\mathbb{C})$ and $X(d X)^{2}$ is a matrix of smooth 2-forms on $\bar{B} U(\mathbb{C})$ and we have

$$
\operatorname{det}\left(I+X(d X)^{2}\right)=\sum_{r \geqslant 0} c_{r}(X)
$$

where $c_{r}(X)$ is a $2 r$-form on $\bar{B} U(\mathbb{C})$.

By using the results above we observe that the forms $c_{r}(X)$ are closed, i.e., $d\left(c_{r}(X)\right)=0$. The class $\frac{1}{(-2 \pi i)^{r}} c_{r}(X)$ is the universal Chern $r$-form and it is well known that

$$
H_{d R}^{*}(\bar{B} U(\mathbb{C})) \stackrel{\simeq}{\longrightarrow} \mathbb{C}\left[c_{1}, c_{2}, \ldots\right]
$$

\section{Zariski closure of manifolds of idempotent matrices}

The aim of this section is to show $G_{n, r}(\mathbb{C})$ is dense in $\operatorname{Idem}_{n, r}(\mathbb{C})$ with respect to the Zariski topology on the affine space $M_{n}(\mathbb{C}) \simeq \mathbb{C}^{n^{2}}$. 
Given a field $K$ and its subfield $k \subseteq K$, relations between affine subsets in $k^{n}$ and their Zariski closures in $K^{n}$ are investigated. We observe that the relative topology on $k^{n}$ induced by the Zariski topology on $K^{n}$ coincides with the Zariski topology on $k^{n}$. Given affine varieties $V \subseteq k^{n}$ and $W \subseteq K^{n}$ with $V \subseteq W$, we make use of [8, Chapter I, Section 6], to show in Proposition 3.4 that $\bar{V}=W$ under some conditions, where $\bar{V}$ denotes the Zariski closure of $V$ in $K^{n}$. In particular, for the subfield $\mathbb{R} \subseteq \mathbb{C}$ of reals we deduce that $\overline{G_{n, r}(\mathbb{C})}=\operatorname{Idem}_{n, r}(\mathbb{C})$.

Let $k\left[T_{1}, \ldots, T_{n}\right]$ be the polynomial ring with coefficients in $k$ and indeterminates $T_{1}, \ldots, T_{n}$. For an ideal $I \subseteq k\left[T_{1}, \ldots, T_{n}\right]$ denote by $V_{k}(I)$ the associated Zariski closed subset in $k^{n}$. If $K$ is another field with $k \subseteq K$ and $I \subseteq k\left[T_{1}, \ldots, T_{n}\right]$ an ideal then $V_{k}(I)=\overline{V_{k}(I)} \cap k^{n}$, where $\overline{V_{k}(I)}$ is the Zariski closure of $V_{k}(I)$ in $K^{n}$. Furthermore, it holds:

Proposition 3.1. Let $k \subseteq K$ be a subfield of a field $K$.

(1) For any ideal $J \subseteq K\left[T_{1}, \ldots, T_{n}\right]$ the intersection $V_{K}(J) \cap k^{n}$ is a Zariski closed subset in $k^{n}$;

(2) if $X \subseteq k^{n}$ and $\bar{X}$ denotes the Zariski closure of $X$ in $K^{n}$ then $\bar{X}=V_{K}(I)$, where $I$ is an ideal in $K\left[T_{1}, \ldots, T_{n}\right]$ generated by some polynomials in $k\left[T_{1}, \ldots, T_{n}\right]$.

Proof. If $X \subseteq k^{n}$ then its Zariski closure $\bar{X}$ in $K^{n}$ is given by $V_{K}(J(X))$, where $J(X)$ is the ideal in $K\left[T_{1}, \ldots, T_{n}\right]$ determined by polynomials vanishing on $X$. Given a polynomial $p$ in $K\left[T_{1}, \ldots, T_{n}\right]$, fix a basis $b_{1}, \ldots, b_{s}$ of the $k$-vector subspace of $K$ generated by its coefficients. Then $p=b_{1} p_{1}+\cdots+b_{s} p_{s}$ with $p_{1}, \ldots, p_{s}$ in $k\left[T_{1}, \ldots, T_{n}\right]$.

For an ideal $J=\left(q_{1}, \ldots, q_{t}\right) \subseteq K\left[T_{1}, \ldots, T_{n}\right]$ consider polynomials

$$
q_{1}^{(1)}, \ldots, q_{1}^{\left(s_{1}\right)}, \ldots, q_{t}^{(1)}, \ldots, q_{t}^{\left(s_{t}\right)}
$$

in $k\left[T_{1}, \ldots, T_{n}\right]$, where $q_{l}^{(1)}, \ldots, q_{l}^{\left(s_{l}\right)}$ are polynomials in $k\left[T_{1}, \ldots, T_{n}\right]$ associated with $q_{l}$ by the procedure above, for $l=1, \ldots, t$. It is clear that

(1): $V_{K}(J) \cap k^{n}=V_{k}\left(q_{1}^{(1)}, \ldots, q_{1}^{\left(s_{1}\right)}, \ldots, q_{t}^{(1)}, \ldots, q_{t}^{\left(s_{t}\right)}\right)$.

Suppose now that $\bar{X}=V_{K}(J(X))$. Then (1) implies that

$$
\bar{X} \subseteq V_{K}\left(q_{1}^{(1)}, \ldots, q_{1}^{\left(s_{1}\right)}, \ldots, q_{t}^{(1)}, \ldots, q_{t}^{\left(s_{t}\right)}\right) .
$$

But clearly

$$
V_{K}\left(q_{1}^{(1)}, \ldots, q_{1}^{\left(s_{1}\right)}, \ldots, q_{t}^{(1)}, \ldots, q_{t}^{\left(s_{t}\right)}\right) \subseteq V_{K}(J(X))
$$

and so we get

$(2): \bar{X}=V_{K}(J(X))=V_{K}\left(q_{1}^{(1)}, \ldots, q_{1}^{\left(s_{1}\right)}, \ldots, q_{t}^{(1)}, \ldots, q_{t}^{\left(s_{t}\right)}\right)$ and the proof is complete.

Thus, we can deduce that $k^{n}$ is a topological subspace of $K^{n}$ with respect to appropriate Zariski topologies, for $k \subseteq K$. To present other relations between Zariski closed subsets in $k^{n}$ and $K^{n}$ we need further investigations.

Corollary 3.2. If a subset $X \subseteq k^{n}$ is irreducible then its Zariski closure $\bar{X}$ in $K^{n}$ is also irreducible.

Proof. Suppose that $\bar{X}=V_{1} \cup V_{2}$ for some Zariski closed subsets $V_{1}, V_{2} \subseteq K^{n}$. Then $X=V_{1} \cap X \cup V_{2} \cap X$ and by Proposition 3.1 the sets $V_{1} \cap k^{n}, V_{2} \cap k^{n}$ are closed in 
$k^{n}$ so $V_{1} \cap X$ and $V_{2} \cap X$ are closed in $X$. But $X$ is irreducible, hence $X=V_{1} \cap X$ or $X=V_{2} \cap X$. Thus $X \subseteq V_{1}$ or $X \subseteq V_{2}$ and, consequently, $\bar{X} \subseteq V_{1}$ or $\bar{X} \subseteq V_{2}$. Finally, $\bar{X}=V_{1}$ or $\bar{X}=V_{2}$ and the result follows.

To state the main result of this section we need to show:

Lemma 3.3. Let $L \subseteq k^{n}$ be a $k$-vector subspace and $\langle L\rangle_{K} \subseteq K^{n}$ the $K$-vector subspace of $K^{n}$ generated by $L$ over $K$. Then $\operatorname{dim}_{k} L=\operatorname{dim}_{K}\langle L\rangle_{K}$.

Proof. Any basis of $L$ over $k$ is a set of generators for the $K$-vector space $\langle L\rangle_{K}$. Let $\alpha_{1}, \ldots, \alpha_{d}$ be linearly independent elements in $L$ over $k$ and $a_{1}, \ldots, a_{d}$ elements in $K$ with $a_{1} \alpha_{1}+\cdots+a_{d} \alpha_{d}=0$. Write $b_{1}, \ldots, b_{s}$ for a basis of the $k$-vector subspace of $K$ generated by $a_{1}, \ldots, a_{d}$. Then $a_{1}=a_{11} b_{1}+\cdots+a_{1 s} b_{s}, \ldots, a_{d}=a_{d 1} b_{1}+\cdots+a_{d s} b_{s}$ for some elements $a_{j}$ in the field $k$ and $i=1, \ldots, d, j=1, \ldots, s$. Thus

$$
\left(a_{11} \alpha_{1}+\cdots+a_{d 1} \alpha_{d}\right) b_{1}+\cdots+\left(a_{1 s} \alpha_{1}+\cdots+a_{d s} \alpha_{d}\right) b_{s}=0 .
$$

Consequently, $a_{11} \alpha_{1}+\cdots+a_{d 1} \alpha_{d}=0, \ldots, a_{1 s} \alpha_{1}+\cdots+a_{d s} \alpha_{d}=0$, hence $a_{i j}=0$ for all $i=1, \ldots, d, j=1, \ldots, s$ and the proof follows.

Given an affine variety $V \subseteq k^{n}$, let $J(V)$ denote the ideal in $k\left[T_{1}, \ldots, T_{n}\right]$ determined by all polynomials vanishing on $V$. Then the tangent space $T_{v} V$ to $V$ at its point $v=\left(v_{1}, \ldots, v_{n}\right)$ is given by zeros of the polynomial $\sum_{i=1}^{n} \frac{\partial f}{\partial T_{i}}(v) T_{i}$, for any $f \in J(V)$.

Write $\operatorname{dim}_{k} V$ for the dimension of an affine variety $V \subseteq k^{n}$. Then by [6, Chapter I, Section 5], there is an inequality $\operatorname{dim}_{k} V \leqslant \operatorname{dim}_{k} T_{v} V$ for any point $v$ in an irreducible variety $V$. Recall that the point $v \in V$ is called simple provided $\operatorname{dim}_{k} V=\operatorname{dim}_{k} T_{v} V$. Now we are in a position to show:

Proposition 3.4. Given fields $k \subseteq K$, let $V \subseteq k^{n}$ and $W \subseteq K^{n}$ be affine varieties. If $W$ is irreducible, $V \subseteq W$ and $\operatorname{dim}_{k} V=\operatorname{dim}_{K} W$ then $\bar{V}=W$, where $\bar{V}$ denotes the Zariski closure of $V$ in $K^{n}$.

Proof. Fix an irreducible component $V_{0}$ of $V \subseteq k^{n}$ with the maximal dimension. Then by Corollary 3.2 its Zariski closure $\bar{V}_{0}$ in the space $K^{n}$ is also irreducible. Let $U \subseteq \bar{V}_{0}$ be the subset given by all simple points in $\bar{V}_{0}$. By [6, Chapter I, Section 5], the set $U$ is open and dense in $\bar{V}_{0}$. Thus $U \cap V_{0} \neq \emptyset$ and by means of Proposition 3.1 and Lemma 3.3 one gets

$\operatorname{dim}_{K} W=\operatorname{dim}_{k} V=\operatorname{dim}_{k} V_{0} \leqslant \operatorname{dim}_{k} T_{v} V_{0}=\operatorname{dim}_{K}\left\langle T_{v} V_{0}\right\rangle \leqslant \operatorname{dim}_{K} T_{v} \bar{V}_{0}=\operatorname{dim}_{K} \bar{V}_{0}$, for any point $v \in U \cap V_{0}$. But $\bar{V}_{0} \subseteq W$ and by [8, Chapter I, Section 6], yields $\operatorname{dim}_{K} \bar{V}_{0} \leqslant \operatorname{dim}_{K} W$. Finally, $\operatorname{dim}_{K} \overline{\bar{V}}_{0}=\operatorname{dim}_{k} V_{0}=\operatorname{dim}_{K} W$ and again by [8, Chapter I, Section 6], we get $\bar{V}_{0}=W$. Then the inclusion $V_{0} \subseteq V \subseteq W$ implies $\bar{V}=W$ and the proof is complete.

Because the Zariski closure preserves Cartesian products, we conclude:

Corollary 3.5. Let $k \subseteq K, V_{1} \subseteq k^{n_{1}}, \ldots, V_{m} \subseteq k^{n_{m}}$ and $W_{1} \subseteq K^{n_{1}}, \ldots, W_{m} \subseteq K^{n_{m}}$ be affine varieties. If $W_{1}, \ldots, W_{m}$ are irreducible, $V_{1} \subseteq W_{1}, \ldots, V_{m} \subseteq W_{m}$ and $\operatorname{dim}_{k} V_{1}=\operatorname{dim}_{K} W_{1}, \ldots, \operatorname{dim}_{k} V_{m}=\operatorname{dim}_{K} W_{m}$ then

$$
\overline{V_{1} \times \cdots \times V_{m}}=\bar{V}_{1} \times \cdots \times \bar{V}_{m}=W_{1} \times \cdots \times W_{m} .
$$


Let $\mathbb{S}_{\mathbb{R}}^{n}$ and $\mathbb{S}_{\mathbb{C}}^{n}$ be the real and complex $n$-spheres, respectively. It is well known that $\mathbb{S}_{\mathbb{C}}^{n}$ is diffeomorphic to the tangent bundle $T \mathbb{S}_{\mathbb{R}}^{n}$ of the sphere $\mathbb{S}_{\mathbb{R}}^{n}$ and coincides with the Zariski closure $\overline{\mathbb{S}_{\mathbb{R}}^{n}}$ in the affine space $\mathbb{C}^{n+1}$. More generally, given a nonsingular real variety $M \subset \mathbb{R}^{n}$ and its Zariski closure $\bar{M}$ in the affine space $\mathbb{C}^{n}$ in the light of Proposition 3.4 we get $\operatorname{dim}_{\mathbb{R}} M=\operatorname{dim}_{\mathbb{C}} \bar{M}$. On the other hand, $M$ can be identified with the image in the tangent bundle $T M$ of the zero cross section. This gives rise to:

Corollary 3.6. Let $M \subseteq \mathbb{R}^{n}$ be a nonsingular real variety. If the tangent bundle $T M$ is a complex variety then the Zariski closure $\bar{M}$ in the affine space $\mathbb{C}^{n}$ coincides with $T M$.

Because $T^{2}-T$ is the minimal polynomial of any nonzero idempotent $n \times n$-matrix over $\mathbb{C}$ hence its rank coincides with the trace. Consequently, the Grassmannian $G_{n, r}(\mathbb{C})$, identified with the idempotent and hermitian $n \times n$-matrices over $\mathbb{C}$ with rank $r$, can be viewed as a real affine variety. Thus $G_{n, r}(\mathbb{C})=\left\{(\varphi, \psi) \in M_{n}(\mathbb{R}) \times M_{n}(\mathbb{R})\right.$; $\left.\operatorname{tr}(\varphi)=r, \varphi^{2}-\psi^{2}=\varphi, \varphi \psi+\psi \varphi=\psi, \varphi^{t}=\varphi, \psi^{t}=-\psi\right\} \subseteq M_{n}(\mathbb{C}) \simeq \mathbb{C}^{n^{2}} \simeq \mathbb{R}^{2 n^{2}}$.

Now consider the complex variety $G_{n, r}^{\mathbb{C}}(\mathbb{C})=\left\{(\varphi, \psi) \in M_{n}(\mathbb{C}) \times M_{n}(\mathbb{C}) ; \operatorname{tr}(\varphi)=r\right.$, $\left.\varphi^{2}-\psi^{2}=\varphi, \varphi \psi+\psi \varphi=\psi, \varphi^{t}=\varphi, \psi^{t}=-\psi\right\} \subseteq \mathbb{C}^{2 n^{2}}$. Then the maps

$$
\mu: G_{n, r}^{\mathbb{C}}(\mathbb{C}) \longrightarrow \operatorname{Idem}_{n, r}(\mathbb{C})
$$

given by $\mu(\varphi, \psi)=\varphi+i \psi$ for any $(\varphi, \psi) \in G_{n, r}^{\mathbb{C}}(\mathbb{C})$ and

$$
\nu: \operatorname{Idem}_{n, r}(\mathbb{C}) \longrightarrow G_{n, r}^{\mathbb{C}}(\mathbb{C})
$$

given by $\nu(\gamma)=\left(\frac{\gamma+\gamma^{t}}{2}, i \frac{-\gamma+\gamma^{t}}{2}\right)$ for any $\gamma \in \operatorname{Idem}_{n, r}(\mathbb{C})$ establish a polynomial isomorphism of complex varieties.

By [8, Chapter II, Section 2], any simple point $x \in X$ in a quasi-projective variety $X$ belongs to a unique irreducible component of $X$. Therefore, any two irreducible components of a smooth quasi-projective variety $X$ coincide or are disjoint.

In particular, any connected smooth quasi-projective variety is irreducible. Because the manifold $\operatorname{Idem}_{n, r}(\mathbb{C})$ is connected in the canonical topology so it is also connected in the Zariski topology and, consequently, irreducible as well.

But $G_{n, r}(\mathbb{C}) \subseteq \operatorname{Idem}_{n, r}(\mathbb{C})$ and $\operatorname{dim}_{\mathbb{R}} G_{n, r}(\mathbb{C})=\operatorname{dim}_{\mathbb{C}} \operatorname{Idem}_{n, r}(\mathbb{C})=\operatorname{dim}_{\mathbb{C}} G_{n, r}^{\mathbb{C}}(\mathbb{C})$ $=2 r(n-r)$ hence, by means of Proposition 3.4 we get $\overline{G_{n, r}(\mathbb{C})}=G_{n, r}^{\mathbb{C}}(\mathbb{C})$. Thus we have the main result of this section:

Theorem 3.7. The manifolds $G_{n, r}(\mathbb{C})$ and $\operatorname{Idem}_{n, r}(\mathbb{C})$ correspond to affine varieties in $\mathbb{R}^{2 n^{2}}$ and $\mathbb{C}^{n^{2}}$, respectively and

$$
\begin{aligned}
\overline{G_{n_{1}, r_{1}}(\mathbb{C}) \times \cdots \times G_{n_{m}, r_{m}}(\mathbb{C})} & =G_{n_{1}, r_{1}}^{\mathbb{C}}(\mathbb{C}) \times \cdots \times G_{n_{m}, r_{m}}^{\mathbb{C}}(\mathbb{C}) \\
& \simeq \operatorname{Idem}_{n_{1}, r_{1}}(\mathbb{C}) \times \cdots \times \operatorname{Idem}_{n_{m}, r_{m}}(\mathbb{C})
\end{aligned}
$$

for any $m, n \geqslant 1$.

In the rest of this paper $K$ is one of the fields: $\mathbb{Q}, \mathbb{R}$ or $\mathbb{C}$.

\section{Universal classes}

Let $X^{n}$ be the polynomial $n \times n$-matrix with entries $X_{p q}$ for $1 \leqslant p, q \leqslant n, K\left[X^{n}\right]$ is the polynomial algebra with indeterminates $X_{p q},\left(\left(X^{n}\right)^{2}-X^{n}, \operatorname{tr} X^{n}-r\right)$ the ideal 
generated by the entries of the matrix $\left(X^{n}\right)^{2}-X^{n}$ together with the polynomial $\operatorname{tr} X^{n}-r$, and we may regard $X^{n}$ as an idempotent matrix of order $n$ with the trace equal to $r$ in the quotient $\operatorname{ring} K\left[X^{n}\right] /\left(\left(X^{n}\right)^{2}-X^{n}, \operatorname{tr} X^{n}-r\right)$. Finally, let $\operatorname{Pol}_{K}\left(\operatorname{Idem}_{n, r}(\mathbb{C})\right)$ be the subalgebra of polynomial functions on $\operatorname{Idem}_{n, r}(\mathbb{C})$ with coefficients in $K$, generated by projection maps $x_{p q}: \operatorname{Idem}_{n, r}(\mathbb{C}) \rightarrow \mathbb{C}$.

Lemma 4.1. The map

$$
K\left[X^{n}\right] /\left(\left(X^{n}\right)^{2}-X^{n}, \operatorname{tr} X^{n}-r\right) \longrightarrow \operatorname{Pol}_{K}\left(\operatorname{Idem}_{n, r}(\mathbb{C})\right)
$$

sending the class of $X_{p q}$ to $x_{p q}$ is a well defined ring isomorphism.

Proof. The map above is clearly well defined. To show that it is an epimorphism, let $P: \operatorname{Idem}_{n, r}(\mathbb{C}) \rightarrow \mathbb{C}$ be a polynomial map with coefficients in $K$, i.e., there exists $\tilde{P} \in K\left[X^{n}\right]$ such that $\tilde{P}(A)=P(A)$ for any $A \in \operatorname{Idem}_{n, r}(\mathbb{C})$. It is obvious that the class of $\tilde{P}$ in $K\left[X^{n}\right] /\left(\left(X^{n}\right)^{2}-X^{n}, \operatorname{tr} X^{n}-r\right)$ is sent to $P$.

To prove the injectivity, suppose that $\tilde{P} \in K\left[X^{n}\right]$ vanishes on $\operatorname{Idem}_{n, r}(\mathbb{C})$ which by Theorem 3.7 is a Zariski closed subset of $M_{n}(\mathbb{C})$, and so $\tilde{P}$ belongs to the ideal $\left(\left(X^{n}\right)^{2}-X^{n}, \operatorname{tr} X^{n}-r\right)$ of the polynomial ring $\mathbb{C}\left[X^{n}\right]$.

Therefore, if $K$ is either $\mathbb{R}$ or $\mathbb{C}$, the polynomial $\tilde{P}$ belongs to the ideal $\left(\left(X^{n}\right)^{2}-\right.$ $\left.X^{n}, \operatorname{tr} X^{n}-r\right)$ of the polynomial ring $K\left[X^{n}\right]$, i.e., the class of $\tilde{P}$ in $K\left[X^{n}\right] /\left(\left(X^{n}\right)^{2}-\right.$ $\left.X^{n}, \operatorname{tr} X^{n}-r\right)$ is zero.

Finally, if $K=\mathbb{Q}$, the canonical isomorphism

$$
\mathbb{Q}\left[X^{n}\right] /\left(\left(X^{n}\right)^{2}-X^{n}, \operatorname{tr} X^{n}-r\right) \otimes_{\mathbb{Q}} \mathbb{R} \rightarrow \mathbb{R}\left[X^{n}\right] /\left(\left(X^{n}\right)^{2}-X^{n}, \operatorname{tr} X^{n}-r\right)
$$

yields that the class of $\tilde{P}$ is zero in $\mathbb{Q}\left[X^{n}\right] /\left(\left(X^{n}\right)^{2}-X^{n}, \operatorname{tr} X^{n}-r\right)$ and the proof follows.

We consider now the non-degenerate $\mathbb{C}$-bilinear map $\{\}:, M_{n}(\mathbb{C}) \times M_{n}(\mathbb{C}) \rightarrow$ $\mathbb{C}$ given by $\{M, N\}=\operatorname{tr}(M N)$. Then $\operatorname{Idem}_{n, r}(\mathbb{C})$ inherits a semiriemannian metric and one shows immediately that for any $A \in \operatorname{Idem}_{n, r}(\mathbb{C})$ the tangent and normal to $\operatorname{Idem}_{n, r}(\mathbb{C})$ at $A$ are given respectively by

$$
\begin{aligned}
T_{A}\left(\operatorname{Idem}_{n, r}(\mathbb{C})\right) & =\left\{B \in M_{n}(\mathbb{C}) \mid A B+B A=B\right\}, \\
N_{A}\left(\operatorname{Idem}_{n, r}(\mathbb{C})\right) & =\left\{B \in M_{n}(\mathbb{C}) \mid A B=B A\right\},
\end{aligned}
$$

and we have the orthogonal decomposition

$$
M_{n}(\mathbb{C})=T_{A} \operatorname{Idem}_{n, r}(\mathbb{C}) \oplus N_{A} \operatorname{Idem}_{n, r}(\mathbb{C})
$$

given by

$$
B=(A B+B A-2 A B A) \oplus(B-A B-B A+2 A B A) .
$$

To sate the next lemma we recall that a polynomial map $f: \operatorname{Idem}_{n, r}(\mathbb{C}) \rightarrow M_{n}(\mathbb{C})$ is called to a normal section if $f(A) A=A f(A)$ fr all $A \in \operatorname{Idem}_{n, r}(\mathbb{C})$.

Lemma 4.2. Let $\Phi=\sum_{\alpha, \beta} P_{\alpha \beta} d x_{\alpha \beta}$ be a 1-form on $M_{n}(\mathbb{C})$ with $P_{\alpha \beta}$ polynomials in $K\left[X^{n}\right]$. Then, its restriction to $\operatorname{Idem}_{n, r}(\mathbb{C})$ is zero if and only if the map $\operatorname{Idem}_{n, r}(\mathbb{C}) \rightarrow M_{n}(\mathbb{C})$, given by $A \rightarrow P(A)^{t}$ is a normal section of $\operatorname{Idem}_{n, r}(\mathbb{C})$, or equivalently if and only if all entries of $P\left(X^{n}\right)^{t}\left(X^{n}\right)-\left(X^{n}\right) P\left(X^{n}\right)^{t}$ belong to the ideal $\left(\left(X^{n}\right)^{2}-X^{n}, \operatorname{tr}\left(X^{n}\right)-r\right)$, where $P\left(X^{n}\right)$ is the $n \times n$-matrix of polynomials whose entry $\alpha \beta$ is $P_{\alpha \beta}$. 
Proof. For any $A, B \in M_{n}(\mathbb{C})$ we have

$$
\Phi_{A}(B)=\sum_{\alpha \beta} P_{\alpha \beta}(A)\left(d x_{\alpha \beta}\right)_{A}(B)=\sum_{\alpha \beta} P_{\alpha \beta}(A) B_{\alpha \beta}=\operatorname{tr}\left(P(A)^{t} B\right)=\left\{P(A)^{t}, B\right\} .
$$

Therefore, $\Phi$ vanishes on $\operatorname{Idem}_{n, r}(\mathbb{C})$ if and only if $\left\{P(A)^{t}, B\right\}=0$ for any $A \in$ $\operatorname{Idem}_{n, r}(\mathbb{C})$ and any $B \in T_{A} \operatorname{Idem}_{n, r}(\mathbb{C})$, i.e., if $P(A)^{t} \in N_{A} \operatorname{Idem}_{n, r}(\mathbb{C})$.

Thus, $\Phi$ vanishes on $\operatorname{Idem}_{n, r}(\mathbb{C})$ if and only if $P(A)^{t} A=A P(A)^{t}$ for all $A \in$ $\operatorname{Idem}_{n, r}(\mathbb{C})$, or equivalently if and only if all entries of the matrix of polynomials $P\left(X^{n}\right)^{t} X^{n}-X^{n} P\left(X^{n}\right)^{t}$ vanish on $\operatorname{Idem}_{n, r}(\mathbb{C})$.

Finally, the proof follows because by Theorem 3.7 the variety $\operatorname{Idem}_{n, r}(\mathbb{C})$ is Zariski closed.

Let $\operatorname{Pol}_{K}\left(\operatorname{Idem}_{n, r}(\mathbb{C}), M_{n}(\mathbb{C})\right)$ be the $\operatorname{Pol}_{K}\left(\operatorname{Idem}_{n, r}(\mathbb{C})\right)$-free module of polynomial maps $f: \operatorname{Idem}_{n, r}(\mathbb{C}) \rightarrow M_{n}(\mathbb{C})$ with coefficients in $K$, and let $\operatorname{Pol}_{K}^{N}\left(\operatorname{Idem}_{n, r}(\mathbb{C})\right.$, $M_{n}(\mathbb{C})$ ) be the submodule of normal polynomial sections on $\operatorname{Idem}_{n, r}(\mathbb{C})$, i.e., polynomial maps $f: \operatorname{Idem}_{n, r}(\mathbb{C}) \rightarrow M_{n}(\mathbb{C})$ such that $f(A) A=A f(A)$ for any $A \in$ $\operatorname{Idem}_{n, r}(\mathbb{C})$, with all entries of $f$ being polynomials with coefficients in $K$.

By using, for any $A \in \operatorname{Idem}_{n, r}(\mathbb{C})$, the orthogonal decomposition above

$$
M_{n}(\mathbb{C})=T_{A} \operatorname{Idem}_{n, r}(\mathbb{C}) \oplus N_{A} \operatorname{Idem}_{n, r}(\mathbb{C}),
$$

we define, for any $B \in M_{n}(\mathbb{C})$, the normal polynomial section

$$
\pi_{N}(B): \operatorname{Idem}_{n, r}(\mathbb{C}) \rightarrow M_{n}(\mathbb{C})
$$

given by $\pi_{N}(B)(A)=B-A B-B A+2 A B A$, and clearly the $n^{2}$ normal polynomial sections $\pi_{N}\left(E^{p q}\right)$ generate the module $\operatorname{Pol}_{K}^{N}\left(\operatorname{Idem}_{n, r}(\mathbb{C}), M_{n}(\mathbb{C})\right)$, where $E^{p q} \in$ $M_{n}(\mathbb{C})$ has the $p q$-entry equal to 1 and all others zero.

Therefore, we have a $\operatorname{Pol}_{K}\left(\operatorname{Idem}_{n, r}(\mathbb{C})\right)$-module epimorphism

$$
\pi_{N}: \operatorname{Pol}_{K}\left(\operatorname{Idem}_{n, r}(\mathbb{C}), M_{n}(\mathbb{C})\right) \rightarrow \operatorname{Pol}_{K}^{N}\left(\operatorname{Idem}_{n, r}(\mathbb{C}), M_{n}(\mathbb{C})\right)
$$

given by

$$
\pi_{N}(f)(A)=f(A)-A f(A)-f(A) A+2 A f(A) A,
$$

and the following lemma holds:

Lemma 4.3. The algebraic de Rham complex of $K\left[X^{n}\right] /\left(\left(X^{n}\right)^{2}-X^{n}, \operatorname{tr} X^{n}-r\right)$ is canonically isomorphic with de Rham complex of polynomial forms on $\operatorname{Idem}_{n, r}(\mathbb{C})$ with coefficients in $K$.

In particular, the algebraic de Rham cohomology of $K\left[X^{n}\right] /\left(\left(X^{n}\right)^{2}-X^{n}\right.$, $\left.\operatorname{tr} X^{n}-r\right)$ is canonically isomorphic to the cohomology of the complex of polynomial forms with coefficients in $K$ over $\operatorname{Idem}_{n, r}(\mathbb{C})$.

Proof. Consider the ring isomorphism

$$
K\left[X^{n}\right] /\left(\left(X^{n}\right)^{2}-X^{n}, \operatorname{tr} X^{n}-r\right) \rightarrow \operatorname{Pol}_{K}\left(\operatorname{Idem}_{n, r}(\mathbb{C})\right)
$$

given by Lemma 4.1. We show that the induced map $\varphi$, from the module of Kähler differentials over $K\left[X^{n}\right] /\left(\left(X^{n}\right)^{2}-X^{n}, \operatorname{tr} X^{n}-r\right)$ to that of polynomial differential 1-forms over $\operatorname{Pol}_{K}\left(\operatorname{Idem}_{n, r}(\mathbb{C})\right)$ with coefficients in $K$ is again an isomorphism.

It is obvious that $\varphi$ is surjective. 
To show injectivity, let $\Phi=\sum_{\alpha \beta} P_{\alpha \beta} d X_{\alpha \beta}$ be such that $\varphi(\Phi)=0$.

By Lemma 4.3 we know that $P\left(X^{n}\right)^{t}$ is a normal section on $\operatorname{Idem}_{n, r}(\mathbb{C})$, and so it is a linear combination of the generators $\pi_{N}\left(E^{p q}\right)$.

Therefore $\Phi$ is a linear combination of the Kähler differentials

$$
\omega_{p q}=\sum_{\alpha \beta}\left(\pi_{N}\left(E^{p q}\right)(X)\right)_{\beta \alpha} d X_{\alpha \beta} .
$$

Because we have

$$
\begin{aligned}
\omega_{p q} & =\sum_{\alpha \beta}\left(E^{p q}-X^{n} E^{p q}-E^{p q} X^{n}+2 X^{n} E^{p q} X^{n}\right)_{\beta \alpha} d X_{\alpha \beta} \\
& =\sum_{\alpha \beta}\left(\delta_{p \beta} \delta_{q \alpha}-X_{\beta p} \delta_{q \alpha}-\delta_{\beta p} X_{q \alpha}+2 X_{\beta p} X_{q \alpha}\right) d X_{\alpha \beta} \\
& =d X_{q p}-\sum_{\beta=1}^{n} X_{\beta p} d X_{q \beta}-\sum_{\alpha=1}^{n} X_{q \alpha} d X_{\alpha p}+2 \sum_{\alpha \beta} X_{\beta p} X_{q \alpha} d X_{\alpha \beta} \\
& =\left(d X^{n}-d X^{n} X^{n}-X^{n} d X^{n}+2 X^{n} d X^{n} X^{n}\right)_{q p} \\
& =\left(d\left(X^{n}-\left(X^{n}\right)^{2}\right)+2 X^{n} d X^{n} X^{n}\right)_{q p}=0
\end{aligned}
$$

the proof follows.

It is well known after Grothendieck [5]:

Lemma 4.4. The canonical map

$$
H_{d R}^{*}\left(\mathbb{C}\left[X^{n}\right] /\left(\left(X^{n}\right)^{2}-X^{n}, \operatorname{tr} X^{n}-r\right)\right) \rightarrow H_{d R}^{*}\left(\operatorname{Idem}_{n, r}(\mathbb{C})\right),
$$

from the algebraic de Rham cohomology of $\mathbb{C}\left[X^{n}\right] /\left(\left(X^{n}\right)^{2}-X^{n}, \operatorname{tr} X^{n}-r\right)$ to de Rham cohomology of $\operatorname{Idem}_{n, r}(\mathbb{C})$ with complex coefficients, is an isomorphism.

Lemmas 4.3 and 4.4, and the standard de Rham isomorphism lead to:

Corollary 4.5. There is a sequence of isomorphisms

$$
\begin{aligned}
H_{d R}^{*}\left(\mathbb{C}\left[X^{n}\right] /\left(\left(X^{n}\right)^{2}-X^{n}, \operatorname{tr} X^{n}-r\right)\right) & \stackrel{\simeq}{\longrightarrow} H_{d R}^{*}\left(\operatorname{Pol}_{\mathbb{C}}\left(\operatorname{Idem}_{n, r}(\mathbb{C})\right)\right) \\
& \simeq H_{d R}^{*}\left(\operatorname{Idem}_{n, r}(\mathbb{C})\right) \stackrel{\simeq}{\longrightarrow} H^{*}\left(\operatorname{Idem}_{n, r}(\mathbb{C}) ; \mathbb{C}\right),
\end{aligned}
$$

where $H_{d R}^{*}\left(\operatorname{Pol}_{\mathbb{C}}\left(\operatorname{Idem}_{n, r}(\mathbb{C})\right)\right)$ denotes the cohomology of the de Rham subcomplex of polynomial differential forms with complex coefficients on $\operatorname{Idem}_{n, r}(\mathbb{C})$ and $H^{*}\left(\operatorname{Idem}_{n, r}(\mathbb{C}) ; \mathbb{C}\right)$ is the singular cohomology with coefficients in $\mathbb{C}$.

As a consequence of Corollary 4.5, together with Lemma 1.2 and the universal coefficient theorem for singular cohomology, we obtain:

Proposition 4.6. There is a sequence of isomorphisms:

$$
\begin{aligned}
H_{d R}^{*}\left(K\left[X^{n}\right] /\left(\left(X^{n}\right)^{2}-X^{n}, \operatorname{tr} X^{n}-r\right)\right) & \stackrel{\simeq}{\longrightarrow} H_{d R}^{*}\left(\operatorname{Pol}_{K}\left(\operatorname{Idem}_{n, r}(\mathbb{C})\right)\right) \\
& \simeq H^{*}\left(\operatorname{Idem}_{n, r}(\mathbb{C}) ; K\right) .
\end{aligned}
$$

The well known cohomology of $H^{*}\left(\operatorname{Idem}_{n, r}(\mathbb{C}) ; K\right)=H^{*}\left(G_{n, r}(\mathbb{C}) ; K\right)$ yields our first main result: 
Theorem 4.7. We have $H_{d R}^{*}\left(K\left[X^{n}\right] /\left(\left(X^{n}\right)^{2}-X^{n}, \operatorname{tr} X^{n}-r\right)\right)=K\left[\bar{c}_{1}, \ldots, \bar{c}_{r}\right] / I_{n, r}$, where $\bar{c}_{j} \in H_{d R}^{2 j}\left(K\left[X^{n}\right] /\left(\left(X^{n}\right)^{2}-X^{n}, \operatorname{tr} X^{n}-r\right)\right)$ is the class of the $j$-th Chern form $c_{j}\left(X^{n}\left(d X^{n}\right)^{2}\right) \in \Omega^{2 j}\left(K\left[X^{n}\right] /\left(\left(X^{n}\right)^{2}-X^{n}, \operatorname{tr} X^{n}-r\right)\right)$ and $I_{n, r}$ is the ideal generated by the well known polynomials deduced from the relation $\left(1+c_{1}(\gamma)+\cdots+c_{r}(\gamma)\right)(1+$ $\left.c_{1}\left(\gamma^{\perp}\right)+\cdots+c_{n-r}\left(\gamma^{\perp}\right)\right)=1$, where $c_{j}(\gamma)$, resp. $c_{j}\left(\gamma^{\perp}\right)$, is the $j$-th Chern class of the canonical bundle $\gamma$, resp. normal to the canonical bundle $\gamma^{\perp}$, over $G_{n, r}(\mathbb{C})$. (see, e.g., [1, Proposition 23.2]).

To illustrate the ideal $I_{n, r}$, we present:

Example 4.8. (1) If $r=1$ then $\left(1+c_{1}(\gamma)\right)\left(1+c_{1}\left(\gamma^{\perp}\right)+\cdots+c_{n-1}\left(\gamma^{\perp}\right)\right)=1$ gives $c_{k}\left(\gamma^{\perp}\right)=(-1)^{k} c_{1}(\gamma)^{k}$ for $k=1, \ldots, n-1$ and $c_{1}(\gamma)^{n}=0$. The ideal $I_{n, 1}$ has a single generator $\bar{c}_{1}^{n}$ and the algebraic de Rham cohomology of $K\left[X^{n}\right] /\left(\left(X^{n}\right)^{2}-X^{n}, \operatorname{tr} X^{n}-\right.$ $1)$ is the quotient $K\left[\bar{c}_{1}\right] /\left(\bar{c}_{1}^{n}\right)$ for $K=\mathbb{Q}, \mathbb{R}$ or $\mathbb{C}$, where $\bar{c}_{1}$ is the class of $\operatorname{tr}\left(X^{n}\left(d X^{n}\right)^{2}\right)$.

(2) If $n=4$ and $r=2$ then $\left(1+c_{1}(\gamma)+c_{2}(\gamma)\right)\left(1+c_{1}\left(\gamma^{\perp}\right)+c_{2}\left(\gamma^{\perp}\right)\right)=1$ gives $c_{1}\left(\gamma^{\perp}\right)=-c_{1}(\gamma), \quad c_{2}\left(\gamma^{\perp}\right)=-c_{2}(\gamma)+c_{1}(\gamma)^{2}, \quad$ and $\quad-2 c_{1}(\gamma) c_{2}(\gamma)+c_{1}(\gamma)^{3}=0$, $-c_{2}(\gamma)^{2}+c_{1}(\gamma)^{2} c_{2}(\gamma)=0$. The ideal $I_{4,2}$ has two generators: $-2 \bar{c}_{1} \bar{c}_{2}+\bar{c}_{1}^{3},-\bar{c}_{2}^{2}+\bar{c}_{1}^{2} \bar{c}_{2}$ and the algebraic de Rham cohomology of $K\left[X^{n}\right] /\left(\left(X^{n}\right)^{2}-X^{n}, \operatorname{tr} X^{n}-2\right)$ is the quotient $K\left[\bar{c}_{1}, \bar{c}_{2}\right] /\left(-2 \bar{c}_{1} \bar{c}_{2}+\bar{c}_{1}^{3},-\bar{c}_{2}^{2}+\bar{c}_{1}^{2} \bar{c}_{2}\right)$ for $K=\mathbb{Q}, \mathbb{R}$ or $\mathbb{C}$.

Lemma 4.9. The induced maps

$$
\lim _{n} K\left[X^{n}\right] /\left(\left(X^{n}\right)^{2}-X^{n}, \operatorname{tr} X^{n}-r\right) \rightarrow \operatorname{Pol}_{K}\left(\operatorname{Idem}_{-, r}(\mathbb{C})\right)
$$

and

$$
\lim _{r}\left(\lim _{n} K\left[X^{n}\right] /\left(\left(X^{n}\right)^{2}-X^{n}, \operatorname{tr} X^{n}-r\right)\right) \rightarrow \operatorname{Pol}_{K}(\bar{B} U(\mathbb{C}))
$$

are ring isomorphisms.

Proof. It is clear that $\operatorname{Pol}_{K}\left(\operatorname{Idem}_{-, r}(\mathbb{C})\right)$ is the inverse $\operatorname{limit}_{\lim _{n}} \operatorname{Pol}_{K}\left(\operatorname{Idem}_{n, r}(\mathbb{C})\right)$

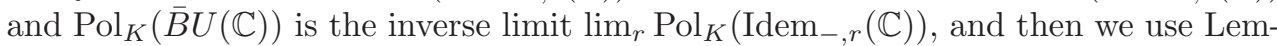
ma 4.1 to complete the proof.

Consider now the following cases:

(1) let $M$ be a topological space and $\cdots M^{n} \subset M^{n+1} \subset \cdots$ an increasing filtration so that $M=\cup_{n} M^{n}$, and such that any compact subset of $M$ is contained in some $M^{n}$. Notice that this condition is always satisfied provided $M$ is a $T_{1}$-space. Denote by $H^{*}(; R)$ singular cohomology with coefficients in a commutative ring $R$;

(2) the spaces $M^{n}$ of the filtration of $M$ are real smooth manifolds, so that $M^{n}$ is a closed smooth submanifold of $M^{n+1}$ for all $n \geqslant 1$; We define then a $p$-form $\Phi$ on $M$ as a sequence of $p$-forms $\Phi_{n}$ on each $M^{n}$ such that $\Phi_{n+1}$ restricts to $\Phi_{n}$ on $M^{n}$. In particular, we obtain a de Rham complex and a corresponding de Rham cohomology for $M$.

(3) The same as above but:

(a) assuming the $M^{n}$ are complex holomorphic manifolds;

(b) considering all $M^{n}$ included in $\mathbb{C}^{\infty}$ and replacing cohomology by de Rham cohomology of complex polynomial forms with coefficients in $K=\mathbb{Q}, \mathbb{R}$ or $\mathbb{C}$.

(4) $\left(\Omega_{n}(\mathcal{A}), \delta_{n}\right)=\left(\Omega_{d R}^{*}\left(K\left[X^{n}\right] /\left(\left(X^{n}\right)^{2}-X^{n}, \operatorname{tr} X^{n}-r\right)\right), d\right)$.

Lemma 4.10. The Mittag-Leffler condition holds in (1), for the case of $M$ being a $C W$-space and $M^{n}$ its n-skeleton, or for cases: (2), (3)(a)-(b) and (4) above. 
Proof. The case of $M$ being a $C W$-space with skeletons $M^{n}$ is standard. Cases (2) and (3)(a) are now deduced by using the standard de Rham theorem.

In order to prove $(3)(b)$ it is enough to show that for any complex holomorphic submanifold $P$ of some $\mathbb{C}^{n}$ the canonical map from the complex of $K$-polynomial forms to the standard de Rham complex, induces an isomorphism in cohomology. But this is well known after Grothendieck [5].

Finally, case (4) is clear by Proposition 4.6 above and the proof is complete.

Now, the following result is an easy consequence of Lemma 1.3, Proposition 4.6, Theorem 4.7, Lemma 4.10 and the required definitions.

Theorem 4.11. There are isomorphisms

$$
\begin{aligned}
H^{*}\left(\operatorname { l i m } _ { n } \left(\Omega_{d R}^{*}(\right.\right. & \left.\left.\left.K\left[X^{n}\right] /\left(\left(X^{n}\right)^{2}-X^{n}, \operatorname{tr} X^{n}-r\right), d\right)\right)\right) \\
& \stackrel{\simeq}{\longrightarrow} \lim _{n} H_{d R}^{*}\left(K\left[X^{n}\right] /\left(\left(X^{n}\right)^{2}-X^{n}, \operatorname{tr} X^{n}-r\right)\right) \\
& \stackrel{\simeq}{\longrightarrow} H_{d R}^{*}\left(\operatorname{Pol}_{K}\left(\operatorname{Idem}_{-, r}(\mathbb{C})\right)\right) \stackrel{\simeq}{\longrightarrow} H^{*}\left(\operatorname{Idem}_{-, r}(\mathbb{C}) ; K\right)=K\left[\bar{c}_{1}, \ldots, \bar{c}_{r}\right],
\end{aligned}
$$

and

$$
\begin{aligned}
H^{*}\left(\lim _{r} \lim _{n}\left(\Omega_{d R}^{*}\left(K\left[X^{n}\right] /\left(\left(X^{n}\right)^{2}-X^{n}, \operatorname{tr} X^{n}-r\right), d\right)\right)\right) \\
\stackrel{\simeq}{\longrightarrow} \lim _{r} \lim _{n} H_{d R}^{*}\left(K\left[X^{n}\right] /\left(\left(X^{n}\right)^{2}-X^{n}, \operatorname{tr} X^{n}-r\right)\right) \\
\stackrel{\simeq}{\longrightarrow} H_{d R}^{*}\left(\operatorname{Pol}_{K}(\bar{B} U(\mathbb{C}))\right) \stackrel{\simeq}{\longrightarrow} H^{*}(\bar{B} U(\mathbb{C}) ; K)=K\left[\bar{c}_{1}, \bar{c}_{2}, \ldots\right] .
\end{aligned}
$$

\section{Final results}

In order to get a new description of the inverse limit

$$
\lim _{n} K\left[X^{n}\right] /\left(\left(X^{n}\right)^{2}-X^{n}, \operatorname{tr} X^{n}-r\right)
$$

of Lemma 4.9 we proceed as follows:

Consider $\rho_{n}: K[[X]] \rightarrow K\left[\left[X^{n}\right]\right]$ the epimorphism sending to zero all the $X_{p q}$ for $p>n$ or $q>n$, and being the identity on $K\left[\left[X^{n}\right]\right]$ and define

$$
\hat{K}[X]=\cap_{n \geqslant 1} \rho_{n}^{-1}\left(K\left[X^{n}\right]\right)
$$

being a subring of $K[[X]]$. In particular, we have

$$
K[X] \subset \hat{K}[X] \subset K[[X]] .
$$

Observe that both inclusions above are strict. For example $\sum_{i=1}^{\infty} X_{11}^{i}$ belongs to $K[[X]]$ but does not belong to $\hat{K}[X]$ and $\operatorname{tr} X=\sum_{i=1}^{\infty} X_{i i}$ belongs to $\hat{K}[X]$ but does not to $K[X]$.

It is clear that

$$
\operatorname{det}(X+I)=1+c_{1}(X)+c_{2}(X)+\cdots \in \hat{K}[X],
$$

because $\rho_{n}(\operatorname{det}(X+I))=\operatorname{det}\left(X^{n}+I_{n}\right) \in K\left[X^{n}\right]$.

Further observe that $\hat{K}[X]$ contains all entries of $X^{2}-X$, and the formal series $\operatorname{tr} X-r$ as well. In particular, we may consider the ideal $\left(X^{2}-X, \operatorname{tr} X-r\right)$ of $\hat{K}[X]$ and then the quotient ring $\hat{K}[X] /\left(X^{2}-X, \operatorname{tr} X-r\right)$. 
The canonical epimorphisms $\rho_{n}: \hat{K}[X] \rightarrow K\left[X^{n}\right]$ being the restrictions of $\rho_{n}$ to $\hat{K}[X]$ lead to epimorphisms

$$
\bar{\rho}_{n}: \hat{K}[X] /\left(X^{2}-X, \operatorname{tr} X-r\right) \rightarrow K\left[X^{n}\right] /\left(\left(X^{n}\right)^{2}-X^{n}, \operatorname{tr} X^{n}-r\right)
$$

and then we get a ring homomorphism

$$
\bar{\rho}: \hat{K}[X] /\left(X^{2}-X, \operatorname{tr} X-r\right) \rightarrow \lim _{n} K\left[X^{n}\right] /\left(\left(X^{n}\right)^{2}-X^{n}, \operatorname{tr} X^{n}-r\right) .
$$

Lemma 5.1. The above homomorphism $\bar{\rho}$ is an isomorphism of rings.

Proof. Denote by $\rho_{n}^{n+1}: K\left[X^{n+1}\right] \rightarrow K\left[X^{n}\right]$ the restriction of $\rho_{n}$ to $K\left[X^{n+1}\right]$ and by $\bar{\rho}_{n}^{n+1}: K\left[X^{n+1}\right] /\left(\left(X^{n+1}\right)^{2}-X^{n+1}, \operatorname{tr} X^{n+1}-r\right) \rightarrow K\left[X^{n}\right] /\left(\left(X^{n}\right)^{2}-X^{n}, \operatorname{tr} X^{n}-r\right)$

the corresponding induced epimorphism in the quotient rings.

(a): $\bar{\rho}$ is surjective:

Let $\left(\left[P_{1}\right],\left[P_{2}\right], \ldots\right)$ be an element of $\lim _{n} K\left[X^{n}\right] /\left(\left(X^{n}\right)^{2}-X^{n}, \operatorname{tr} X^{n}-r\right)$, i.e., $\left[P_{n}\right]$ is the class in $K\left[X^{n}\right] /\left(\left(X^{n}\right)^{2}-X^{n}, \operatorname{tr} X^{n}-r\right)$ of $P_{n} \in K\left[X^{n}\right]$ and $\bar{\rho}_{n}^{n+1}\left(\left[P_{n+1}\right]\right)=$ $\left[P_{n}\right]$ for all $n \geqslant 1$.

We show first that we can choose representatives $P_{n} \in K\left[X^{n}\right]$ such that $\rho_{n}^{n+1}\left(P_{n+1}\right)=P_{n}$ for all $n \geqslant 1$.

In fact, $\rho_{1}^{2}\left(P_{2}\right)-P_{1}=S_{1} \in\left(\left(X^{1}\right)^{2}-X^{1}, \operatorname{tr} X^{1}-r\right)$.

Modify $P_{2}$ by taking $P_{2}^{\prime}=P_{2}-S_{1}=P_{1}$, and so $\rho_{1}^{2}\left(P_{2}^{\prime}\right)=P_{1}$.

Modify $P_{3}$ as follows:

$$
\rho_{2}^{3}\left(P_{3}\right)-P_{2}^{\prime}=S_{2} \in\left(\left(X^{2}\right)^{2}-X^{2}, \operatorname{tr} X^{2}-r\right),
$$

and then take $P_{3}^{\prime}=P_{3}-S_{2}$, so that $\rho_{2}^{3}\left(P_{3}^{\prime}\right)=P_{2}^{\prime}$, and so on.

Finally, define

$$
P=P_{1}+\left(P_{2}^{\prime}-P_{1}\right)+\left(P_{3}^{\prime}-P_{2}^{\prime}\right)+\cdots \in K[[X]] .
$$

But $P \in \hat{K}[X]$, because $\rho_{n}(P)=P_{n}^{\prime}$, and so we have $\bar{\rho}([P])=\left(\left[P_{1}\right],\left[P_{2}\right], \ldots\right)$.

(b): $\bar{\rho}$ is injective:

Let $P \in \hat{K}[X]$ be such that $\rho_{n}(P)=P_{n} \in\left(\left(X^{n}\right)^{2}-X^{n}, \operatorname{tr} X^{n}-r\right)$ for $n \geqslant 1$. We want to show that $P$ belongs to the ideal $\left(X^{2}-X, \operatorname{tr} X-r\right)$ of the $\operatorname{ring} \hat{K}[X]$.

There exist polynomials $F_{p q}^{n} \in K\left[X^{n}\right], 1 \leqslant p, q \leqslant n$, and $F_{n} \in K\left[X^{n}\right]$ such that

$$
P_{n}=\sum_{1 \leqslant p, q \leqslant n} F_{p q}^{n} \rho_{n}\left(\left(X^{2}-X\right)_{p q}\right)+F_{n} \rho_{n}(\operatorname{tr} X-r) .
$$

Define inductively polynomials $\bar{F}_{p q}^{n}$ and $\bar{F}_{n}$ in $K\left[X^{n}\right], 1 \leqslant p, q \leqslant n$ as follows: $\bar{F}_{11}^{1}=$ $F_{11}^{1}, \bar{F}_{1}=F_{1}$.

If we already have defined $F_{p q}^{m}$ and $\bar{F}_{m}$ for $1 \leqslant p, q \leqslant m, m \leqslant n$, such that $\rho_{m-1}^{m}\left(\bar{F}_{p q}^{m}\right)=\bar{F}_{p}^{m-1} q$ for $1 \leqslant p, q \leqslant m-1$ and $\rho_{m-1}^{m}\left(\bar{F}_{m}\right)=\bar{F}_{m-1}$ then we define $\bar{F}_{p q}^{n+1}=F_{p q}^{n+1}$ if $p=n+1 \quad$ or $\quad q=n+1 ; \quad \bar{F}_{p q}^{n+1}=F_{p q}^{n+1}-\rho_{n}^{n+1}\left(F_{p q}^{n+1}\right)+\bar{F}_{p q}^{n} \quad$ if $1 \leqslant p, q \leqslant n ; \bar{F}_{n+1}=F_{n+1}-\rho_{n}^{n+1}\left(F_{n+1}\right)+\bar{F}_{n}$.

Thus we have $\rho_{n}^{n+1}\left(\bar{F}_{p q}^{n+1}\right)=\bar{F}_{p q}^{n}$ for $1 \leqslant p, q \leqslant n$ and $\rho_{n}^{n+1}\left(\bar{F}_{n+1}\right)=\bar{F}_{n}$.

Next define

$$
\bar{P}_{n}=\sum_{1 \leqslant p, q \leqslant n} \bar{F}_{p q}^{n} \rho_{n}\left(X^{2}-X\right)_{p q}+\bar{F}_{n} \rho_{n}(\operatorname{tr} X-r),
$$

for which we have $\rho_{n}^{n+1}\left(\bar{P}_{n+1}\right)=\bar{P}_{n}, n \geqslant 1$. 
Define $\bar{F}_{p q} \in \hat{K}[X]$ by $\rho_{n}\left(\bar{F}_{p q}\right)=\bar{F}_{p q}^{n} \in K\left[X^{n}\right]$ for $1 \leqslant p, q \leqslant n$ and $\bar{F} \in \hat{K}[X]$ given by $\rho_{n}(\bar{F})=\bar{F}_{n} \in K\left[X^{n}\right]$.

Finally, for $\bar{P}=\sum_{p q} \bar{F}_{p q}\left(X^{2}-X\right)_{p q}+\bar{F}(\operatorname{tr} X-r)$ it holds $\rho_{n}(\bar{P})=\bar{P}_{n}$.

Observe that $\rho_{n}(P)-\rho_{n}(\bar{P}) \in\left(\left(X^{n}\right)^{2}-X^{n}, \operatorname{tr} X^{n}-r\right)$ for $n \geqslant 1$. Therefore $P-$ $\bar{P} \in\left(X^{2}-X, \operatorname{tr} X-r\right)$ and so $P=\bar{P}+(P-\bar{P}) \in\left(X^{2}-X, \operatorname{tr} X-r\right)$.

This completes the proof of the injectivity.

Remark 5.2. The quotient $\hat{K}[X] /\left(X^{2}-X, \operatorname{tr} X-r\right)$ is the completion of $K[X] /\left(X^{2}-\right.$ $X, \operatorname{tr} X-r)$ with respect to the decreasing sequence of ideals

$$
\cdots \supset \operatorname{ker} \bar{\rho}_{n} \supset \operatorname{ker} \bar{\rho}_{n+1} \supset \cdots,
$$

where $\bar{\rho}_{n}: K[[X]] /\left(X^{2}-X, \operatorname{tr} X-r\right) \rightarrow K\left[X^{n}\right] /\left(\left(X^{n}\right)^{2}-X^{n}, \operatorname{tr} X^{n}-r\right)$ is induced by the epimorphism $\rho_{n}: K[[X]] \rightarrow K\left[\left[X^{n}\right]\right]$ given at the beginning of this section.

In fact, $\hat{K}[X]$ is the completion of $K[X]$ in $K[[X]]$ with respect to the decreasing sequence of ideals $\cdots \supset \operatorname{ker} \rho_{n} \supset \operatorname{ker} \rho_{n+1} \supset \cdots$, and then we use [7, Chapter 9, Proposition 23.D].

Similarly $\mathrm{Pol}_{K}\left(\operatorname{Idem}_{-, r}(\mathbb{C})\right)$ is complete with respect to the sequence of ideals

$$
\cdots \supset I_{n} \supset I_{n+1} \supset \cdots,
$$

where $I_{n}$ consists of those $K$-polynomials on $\operatorname{Idem}_{-, r}(\mathbb{C})$ which vanish on $\operatorname{Idem}_{n, r}(\mathbb{C})$.

Furthermore, the de Rham complex $\Omega_{\mathrm{Pol}_{K}}^{*}\left(\operatorname{Idem}_{-, r}(\mathbb{C})\right)$ of polynomial forms on Idem $_{-, r}(\mathbb{C})$ with coefficients in $K$ is complete with respect to the decreasing sequence of ideals

$$
\cdots \supset I_{n}^{*} \supset I_{n+1}^{*} \supset \cdots,
$$

where $I_{n}^{*}$ consists of those $K$-polynomial forms on $\operatorname{Idem}_{-, r}(\mathbb{C})$ vanishing on $\operatorname{Idem}_{n, r}(\mathbb{C})$.

Let

$$
\Omega^{*}(K[[X]])=\oplus_{s \geqslant 0} \Omega^{s}(K[[X]]),
$$

where $\Omega^{0}(K[[X]])=K[[X]]$ and, for $s \geqslant 1, \Omega^{s}(K[[X]])$ consists of the formal sums

$$
\Phi=\sum_{p_{1}, q_{1}, \ldots, p_{s}, q_{s}} \Phi_{p_{1} q_{1} \ldots p_{s} q_{s}} d X_{p_{1} q_{1}} \ldots d X_{p_{s} q_{s}}
$$

with $\Phi_{p_{1} q_{1} \ldots p_{s} q_{s}} \in K[[X]]$ and such that

$$
d X_{p_{\sigma 1} q_{\sigma 1}} \ldots d X_{p_{\sigma s} q_{\sigma s}}=\epsilon_{\sigma} d X_{p_{1} q_{1}} \ldots d X_{p_{s} q_{s}}
$$

where $\epsilon_{\sigma}$ is the sign of the permutation $\sigma$ of the set $\{1, \ldots, s\}$.

We have inclusions

$$
\Omega_{K}^{*}\left(K\left[X^{n}\right]\right) \subset \Omega_{K}^{*}(K[X]) \subset \Omega^{*}(K[[X]]) .
$$

Notice that the epimorphisms $\rho_{n}$ at the beginning of this section extend to epimorphisms $\rho_{n}: \Omega^{*}(K[[X]]) \rightarrow \Omega_{K}^{*}\left(K\left[X^{n}\right]\right)$ and define

$$
\hat{\Omega}^{*}(\hat{K}[X])=\cap_{n \geqslant 1} \rho_{n}^{-1}\left(\Omega_{K}^{*}\left(K\left[X^{n}\right]\right)\right) .
$$

In particular, observe that $\hat{\Omega}^{0}(K[[X]])=\hat{K}[X]$ and that $\hat{\Omega}^{*}(K[[X]])$ is the completion of $\Omega_{K}^{*}(K[X])$ with respect to the decreasing chain of ideals

$$
\cdots \supset \operatorname{ker} \rho_{n} \supset \operatorname{ker} \rho_{n+1} \supset \cdots .
$$


We have a canonical derivation

$$
d: \hat{K}[X] \rightarrow \hat{\Omega}^{1}(K[[X]])
$$

given by $d P=\sum_{p q} \frac{\partial P}{\partial X_{p q}} d X_{p q}$ for any $P \in K[[X]]$, and its natural extension to a differential of degree 1 on $\hat{\Omega}^{*}(K[[X]])$.

Observe that $\hat{\Omega}^{*}(K[[X]])$ does not coincide with the $K[[X]]$-module $\Omega_{K}(K[[X]])$ of Kähler differentials of $K[[X]]$, i.e., the unique homomorphism of modules $\varphi: \Omega_{K}(K[[X]]) \rightarrow \hat{\Omega}^{1}(K[[X]])$ such that $\varphi \circ d=d$, given by the universal property, is not an isomorphism.

Define $\hat{\Omega}^{*}\left(\hat{K}[X] /\left(X^{2}-X, \operatorname{tr} X-r\right)\right)$ as the quotient of $\hat{\Omega}^{*}(K[[X]])$ by the ideal generated by $X^{2}-X, \operatorname{tr} X-r, d X X+X d X-d X, \operatorname{tr} d X$. It is clear that the differential $d$ in $\hat{\Omega}^{*}(K[[X]])$ induces a differential in the quotient $\hat{\Omega}^{*}\left(\hat{K}[X] /\left(X^{2}-X\right.\right.$, $\operatorname{tr} X-r))$.

The epimorphisms $\rho_{n}: \hat{\Omega}^{*}(K[[X]]) \rightarrow \Omega_{K}^{*}\left(K\left[X^{n}\right]\right)$ yield epimorphisms

$$
\bar{\rho}_{n}: \hat{\Omega}^{*}\left(\hat{K}[X] /\left(X^{2}-X, \operatorname{tr} X-r\right)\right) \rightarrow \Omega_{K}^{*}\left(K\left[X^{n}\right] /\left(\left(X^{n}\right)^{2}-X^{n}, \operatorname{tr} X^{n}-r\right)\right)
$$

and we get an isomorphism of cochain complexes

$$
\bar{\rho}: \hat{\Omega}^{*}\left(\hat{K}[X] /\left(X^{2}-X, \operatorname{tr} X-r\right)\right) \rightarrow \lim _{n} \Omega_{K}^{*}\left(K\left[X^{n}\right] /\left(\left(X^{n}\right)^{2}-X^{n}, \operatorname{tr} X^{n}-r\right)\right) .
$$

Observe that

$$
\hat{\Omega}^{*}\left(\hat{K}[X] /\left(X^{2}-X, \operatorname{tr} X-r\right)\right)=\cap_{n \geqslant 1} \bar{\rho}_{n}^{-1}\left(\Omega_{K}^{*}\left(K\left[X^{n}\right] /\left(\left(X^{n}\right)^{2}-X^{n}, \operatorname{tr} X^{n}-r\right)\right)\right)
$$

is the completion of $\Omega_{K}^{*}\left(K[X] /\left(X^{2}-X, \operatorname{tr} X-r\right)\right)$ with respect to the decreasing set of ideals

$$
\cdots \supset \operatorname{ker} \bar{\rho}_{n} \supset \operatorname{ker} \bar{\rho}_{n+1} \supset \cdots .
$$

Then, Theorem 4.11 yields our second main result:

Theorem 5.3. There is a canonical isomorphism

$$
\hat{\Omega}^{*}\left(\hat{K}[X] /\left(X^{2}-X, \operatorname{tr} X-r\right)\right) \rightarrow \Omega_{\mathrm{Pol}_{K}}^{*}\left(\operatorname{Idem}_{-, r}(\mathbb{C})\right)
$$

and then an isomorphism

$$
H^{*}\left(\hat{\Omega}^{*}\left(\hat{K}[X] /\left(X^{2}-X, \operatorname{tr} X-r\right)\right), d\right) \stackrel{\simeq}{\longrightarrow} H^{*}\left(\Omega_{\mathrm{Pol}_{K}}^{*}\left(\operatorname{Idem}_{-, r}(\mathbb{C})\right), d\right)=K\left[\bar{c}_{1}, \ldots, \bar{c}_{r}\right] .
$$

Define now a ring homomorphism $\theta: K[[X]] \rightarrow K[[X]]$ given by $\theta\left(X_{p q}\right)=\delta_{p q}$ if $p=1$ or $q=1$ and $\theta\left(X_{p q}\right)=X_{p-1, q-1}$ if $p \geqslant 2$ and $q \geqslant 2$. Observe that it sends the matrix $X$ to $\left(\begin{array}{ll}1 & 0 \\ 0 & X\end{array}\right)$ and it restricts to a ring homomorphism $\theta: \hat{K}[X] \rightarrow \hat{K}[X]$.

On the other hand, it is clear that $\theta(\operatorname{tr} X-(r+1))=\operatorname{tr} X-r$ for any $r \geqslant 1$, and $\theta\left(X^{2}-X\right)_{p q}=\left(X^{2}-X\right)_{p-1, q-1}$, if $p \geqslant 2$ and $q \geqslant 2 ; \theta\left(X^{2}-X\right)_{p q}=0$, if $p=1$ or $q=1$.

Therefore, we have ring epimorphisms

$$
\theta_{r}^{r+1}: \hat{K}[X] /\left(X^{2}-X, \operatorname{tr} X-(r+1)\right) \rightarrow \hat{K}[X] /\left(X^{2}-X, \operatorname{tr} X-r\right) .
$$

Further observe that inclusions $\tau_{r}: \operatorname{Idem}_{-, r}(\mathbb{C}) \hookrightarrow \operatorname{Idem}_{-, r+1}(\mathbb{C})$ given in Section 1 
yield epimorphisms

$$
\tau_{r}^{*}: \operatorname{Pol}_{K}\left(\operatorname{Idem}_{-, r+1}(\mathbb{C})\right) \rightarrow \operatorname{Pol}_{K}\left(\operatorname{Idem}_{-, r}(\mathbb{C})\right)
$$

and the following diagram commutes:

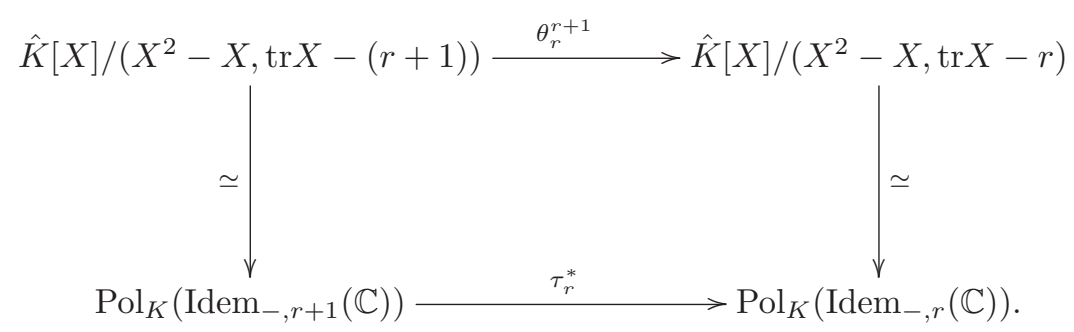

Thus, we have isomorphisms

$$
\begin{aligned}
& \hat{K}[X] /\left(X^{2}-X\right)=\lim _{r} \hat{K}[X] /\left(X^{2}-\right.X, \operatorname{tr} X-r) \\
& \stackrel{\simeq}{\longrightarrow} \lim _{r} \operatorname{Pol}_{K}\left(\operatorname{Idem}_{-, r}(\mathbb{C})\right)=\operatorname{Pol}_{K}(\bar{B} U(\mathbb{C})) .
\end{aligned}
$$

The diagram above extend to the corresponding diagram of commutative graded differential algebras and so we have isomorphisms

$$
\begin{aligned}
\left(\hat{\Omega}^{*}\left(\hat{K}[X] /\left(X^{2}-X\right)\right), d\right)=\left(\operatorname { l i m } _ { r } \hat { \Omega } ^ { * } \left(\hat{K}[X] /\left(X^{2}-X, \operatorname{tr} X\right.\right.\right. & -r)), d) \\
& \stackrel{\simeq}{\longrightarrow}\left(\Omega_{\mathrm{Pol}_{K}}^{*}(\bar{B} U(\mathbb{C})), d\right) .
\end{aligned}
$$

Observe that $\Omega_{\mathrm{Pol}_{K}}^{*}(\bar{B} U(\mathbb{C}))=\lim _{r} \Omega_{\mathrm{Pol}_{K}}^{*}\left(\operatorname{Idem}_{-, r}(\mathbb{C})\right)$ is complete with respect to the decreasing sequence of ideals

$$
\cdots \supset J_{r}^{*} \supset J_{r+1}^{*} \supset \cdots,
$$

where $J_{r}^{*}$ consists of those $K$-polynomial forms on $\bar{B} U(\mathbb{C})$ which vanish on $\operatorname{Idem}_{-, r}(\mathbb{C})$. Consequently, we obtain our third main result:

Theorem 5.4. There is a canonical isomorphism

$$
\hat{\Omega}^{*}\left(\hat{K}[X] /\left(X^{2}-X\right)\right) \rightarrow \Omega_{\mathrm{Pol}_{K}}^{*}(\bar{B} U(\mathbb{C}))
$$

which determines an isomorphism

$$
H^{*}\left(\hat{\Omega}^{*}\left(\hat{K}[X] /\left(X^{2}-X\right)\right)\right) \stackrel{\simeq}{\longrightarrow} H^{*}\left(\Omega_{\mathrm{Pol}_{K}}^{*}(\bar{B} U(\mathbb{C}))\right)=K\left[\bar{c}_{1}, \bar{c}_{2}, \ldots\right] .
$$

Remark 5.5. It is clear that all our main results:

$$
\begin{aligned}
& K\left[T_{1}, \ldots, T_{r}\right] / I_{n, r} \stackrel{\simeq}{\longrightarrow} H_{d R}^{*}\left(K\left[X^{n}\right] /\left(\left(X^{n}\right)^{2}-X^{n}, \operatorname{tr} X^{n}-r\right)\right),(\text { Theorem } 4.7) ; \\
& K\left[T_{1}, \ldots, T_{r}\right] \stackrel{\simeq}{\longrightarrow} H^{*}\left(\lim _{n} \Omega_{K}^{*}\left(K\left[X^{n}\right] /\left(\left(X^{n}\right)^{2}-X^{n}, \operatorname{tr} X^{n}-r\right)\right)\right) \\
& \stackrel{\simeq}{\simeq} H^{*}\left(\hat{\Omega}^{*}\left(\hat{K}[X] /\left(X^{2}-X, \operatorname{tr} X-r\right)\right), d\right),(\text { Theorems } 4.11 \text { and } 5.3) ; \\
& K\left[T_{1}, T_{2}, \ldots\right] \stackrel{\simeq}{\longrightarrow} H^{*}\left(\lim _{r} \lim _{n} \Omega_{K}^{*}\left(K\left[X^{n}\right] /\left(\left(X^{n}\right)^{2}-X^{n}, \operatorname{tr} X^{n}-r\right)\right)\right) \\
& \stackrel{\simeq}{\longleftarrow} H^{*}\left(\hat{\Omega}^{*}\left(\hat{K}[X] /\left(X^{2}-X\right)\right), d\right)(\text { Theorems } 4.11 \text { and } 5.4)
\end{aligned}
$$

hold for any field of characteristic zero and, being theorems in Algebra, it would be interesting giving proofs that use algebraic tools only. 
Remark 5.6. Observe that by using the universal property of Kähler differentials and Theorems 5.3 and 5.4 we get graded ring epimorphisms

$$
\begin{gathered}
H_{d R}^{*}\left(\hat{K}[X] /\left(X^{2}-X, \operatorname{tr} X-r\right)\right) \rightarrow K\left[\bar{c}_{1}, \ldots, \bar{c}_{r}\right], \\
H_{d R}^{*}\left(K[[X]] /\left(X^{2}-X, \operatorname{tr} X-r\right)\right) \rightarrow K\left[\bar{c}_{1}, \ldots, \bar{c}_{r}\right], \\
H_{d R}^{*}\left(\hat{K}[X] /\left(X^{2}-X\right)\right) \rightarrow K\left[\bar{c}_{1}, \bar{c}_{2}, \ldots\right] \text { and } H_{d R}^{*}\left(K[X] /\left(X^{2}-X\right)\right) \rightarrow K\left[\bar{c}_{1}, \bar{c}_{2}, \ldots\right]
\end{gathered}
$$

sending the classes of $c_{p}\left(X(d X)^{2}\right)$ to $\bar{c}_{p}$.

Therefore, the algebraic de Rham cohomology algebras of $\hat{K}[X] /\left(X^{2}-X, \operatorname{tr} X-\right.$ $r), K[[X]] /\left(X^{2}-X, \operatorname{tr} X-r\right)$ (resp. of $\hat{K}[X] /\left(X^{2}-X\right)$ and $\left.K[[X]] /\left(X^{2}-X\right)\right)$ contain a polynomial algebra $K\left[\bar{c}_{1}, \ldots, \bar{c}_{r}\right]$ (resp. $K\left[\bar{c}_{1}, \bar{c}_{2}, \ldots\right]$ ) as a direct summand.

We presume that the actual algebraic de Rham cohomology algebras above are quite complicated.

\section{References}

[1] R. Bott, L.W. Tu, "Differential Forms in Algebraic Topology", Springer-Verlag, New York Inc. (1982).

[2] M. Golasiński, F. Gómez Ruiz, Polynomial and regular maps into Grassmannians, K-Theory 26(1) (2002), 51-68.

[3] M. Golasiński, F. Gómez Ruiz, On Grassmannians over *-rings, Linear Algebra Appl. 355 (2002), 161-172.

[4] F. Gómez Ruiz, Algebraic characteristic classes for idempotent matrices, Publ. Mat. 36 (1992), 601-608.

[5] A. Grothendieck, On the de Rham cohomology of algebraic varieties, Publ. Math. Inst. Houtes Études Sci. 29 (1966), 95-103.

[6] J.E. Humphreys, "Linear Algebraic Groups", Springer-Verlag (1975).

[7] H. Matsumura, "Commutative Algebra", second edition, Benjamin (1980).

[8] I.R. Shafarevich, "Basic Algebraic Geometry. 1. Varieties in Projective Space", Springer-Verlag, Berlin (1994) (translated from the 1988 Russian edition).

[9] R. Swan, Vector bundles and projective modules, Trans. Amer. Math. Soc. 105 (1962), 264-277.

[10] C.A. Weibel, "An Introduction to Homological Algebra", Cambridge University Press (1994).

Marek Golasiński marekg@matman.uwm.edu.pl

Faculty of Mathematics and Computer Science, University of Warmia and Mazury, Słoneczna 54 Street, 10-710 Olsztyn, Poland

Francisco Gómez Ruiz gomez_ruiz@uma.es

Departamento de Álgebra, Geometría y Topología, Facultad de Ciencias, Universidad de Málaga, Campus Universitario de Teatinos, 29071 Málaga, España 